\title{
Antibody Therapies for Large B-Cell Lymphoma
}

\author{
Mattia Novo' \\ Elisa Santambrogio ${ }^{2}$ \\ Pio Manlio Mirko Frascione 3,4 \\ Delia Rota-Scalabrini ${ }^{1}$ \\ Umberto Vitolo' \\ 'Multidisciplinary Oncology Outpatient \\ Clinic, Candiolo Cancer Institute, FPO- \\ IRCCS, Torino, Italy; ${ }^{2} \mathrm{Hematology}$ Unit, \\ Santa Croce e Carle Hospital, Cuneo, \\ Italy; ${ }^{3}$ Medical Oncology, Candiolo \\ Cancer Institute, FPO-IRCCS, Torino, \\ Italy; ${ }^{4}$ Department of Oncology, \\ University of Turin, Torino, Italy
}

Correspondence: Mattia Novo Multidisciplinary Oncology Outpatient Clinic, Candiolo Cancer Institute, FPOIRCCS, Strada Provinciale I42 km 3.95, Candiolo, 10060, Italy

Tel +39 0l I-993-3250

Fax +3901I-9333275

Email mattia.novo@ircc.it

\begin{abstract}
Large B-cell lymphomas (LBCLs) constitute a subgroup of aggressive but highly curable lymphoproliferative diseases. Treatment of relapsed/refractory (R/R) patients still represents an unmet clinical need, and novel drugs and combinations are in continuous development. The pan-B cell panel of surface antigens that characterizes LBCL leads to a large umbrella of druggable targets. Monoclonal antibodies (mAbs) express their activity against lymphoma by targeting multiple tumor-specific antigens. This category consists of a number of molecules with different mechanisms of action, including naked $\mathrm{mAbs}$, radioimmunoconjugates, antibody-drug conjugates, checkpoint inhibitors, and bispecific antibodies. In the last decade, apart from the well-known role of the anti-CD20 mAb rituximab, novel mAbs have led to remarkable steps forward in the treatment of R/R LBCL in monotherapy and combined with chemotherapy. Multiple studies are in development trying to bring these novel compounds into the frontline setting to empower the RCHOP effect or as alternative chemotherapy-free options for elderly/unfit patients. This review provides insight into antilymphoma mAbs, focused on the efficacy and safety of the main molecules approved or in development for LBCL andperspectives on the treatment of this disease.
\end{abstract}

Keywords: diffuse large B-cell lymphoma, immunotherapy, targeted therapy, monoclonal antibodies, checkpoint inhibitors, bispecific antibodies

\section{Introduction}

Diffuse large B-cell lymphoma (DLBCL) represents the most frequent histological subtype of B-cell non-Hodgkin lymphoma (B-NHL) in adults, affecting particularly males and older people. ${ }^{1-3}$ DLBCL is a clinically and biologically heterogeneous disease typically characterized by pan-B-cell surface antigens, such as CD19, CD22, CD22, and CD79A/B, which can be considered potentially druggable targets. ${ }^{1}$ A major milestone in DLBCL treatment has been the introduction of the anti-CD20 monoclonal antibody (mAb) rituximab, and its frontline addition to the standard chemotherapy combination of cyclophosphamide, doxorubicin, vincristine, and prednisone (R-CHOP) led to a cure rate around $60 \%-70 \%$ of cases. ${ }^{4-6}$ Nevertheless, $30 \%-40 \%$ of DLBCL patients relapse and $10 \%$ are refractory to R-CHOP, and the prognosis of this subgroup is usually unfavorable. $^{7,8}$

A less frequent B-NHL belonging to the LBCL category is primary mediastinal B-cell lymphoma (PMBCL), which is now recognized as a unique clinical and biological entity. ${ }^{1}$ It occurs mainly in young adults, with a female predominance, and typically presents as a bulky mediastinal mass. ${ }^{9}$ Despite its particular biological features, overlapping between B-NHL and classical HL, PMBCL expresses the same pan-B-cell targetable antigens of DLBCL (CD19, CD22, CD22, and CD79A/ 
B). The frontline approach for PMBCL has not been standardized yet, but generally consists of rituximab plus an anthracycline-based regimen with or without radiotherapy consolidation. ${ }^{10,11}$ PMBCL has a high cure rate, with long-term progression-free survival (PFS) of 70\%-93\% with different regimens. Nevertheless, $10 \%-30 \%$ of cases are refractory or eventually relapse within $12-18$ months after first-line therapy, with consequent unfavorable outcomes. ${ }^{8,12-20}$

Effective treatment for $\mathrm{R} / \mathrm{R}$ LBCL represents an unmet clinical need. Several biological compounds have been developed to ameliorate outcomes for untreated and $\mathrm{R} / \mathrm{R}$ LBCL, and mAbs are protagonists in this field. This review focuses on mAbs approved or under investigation for treatment of LBCL. A literature search was performed for papers up to February 2021 on PubMed, National Comprehensive Cancer Network and European Society of Medical Oncology guidelines and abstracts from main international conference proceedings, such as American Society of Hematology, American Society of Clinical Oncology, and European Hematology Association meetings. Safety and efficacy data of mAbs derived from the studies on LBCL cited in the manuscript are detailed in Tables 1-3.

\section{Naked Monoclonal Antibodies}

mAbs bind to a specific cell target, inducing tumor-cell death by direct effects on tumor-cell signaling, complement-dependent cytotoxicity, Ab-dependent cytotoxicity, and indirect modulation of the tumor microenvironment. This antineoplastic effect is mediated both by the connection of mAbs with complement, which induces phagocytosis, cytotoxicity, and direct activation of the membrane attack complex, and by interaction with immunoeffector cells, which increases proinflammatory cytokines and cell killing. $^{21}$

Rituximab was the first and is the most commonly used $\mathrm{Ab}$ in the treatment of lymphoproliferative diseases, due to its dramatic results in improving survival. ${ }^{22}$ Since its approval, many combinations with other drugs have been studied, and nowadays biosimilar rituximab compounds and subcutaneous fixed-dose formulations have been approved, with lowering of costs and simplified and shortened drug preparation and administration, respectively. ${ }^{23}$ However, in the last 10 years other similar Abs have been developed in an effort to improve treatment response and outcomes in lymphoproliferative malignancies (Figure 1, Table 1).

\section{Obinutuzumab}

Another anti-CD20 moAb, widely used in lymphoproliferative disorders is obinutuzumab (GA101), a glycolengineered, humanized anti-CD20 Ab with higher antitumor activity than rituximab. Due to the encouraging results demonstrated in follicular lymphoma (FL), GA101 has also been tested for DLBCL. The GAUGUIN trial analyzed the efficacy and safety of single-agent GA101 at different doses for R/R DLBCL, and mantle-cell lymphoma. GA101 showed an overall response rate (ORR) of $32 \%$ in DLBCL., and the most common adverse events (AEs) were infusion-related reactions (IRRs) and neutropenia, which were all manageable. ${ }^{24}$ Led by these data, a large phase III trial (GOYA) on 1,418 untreated DLBCL patients compared GA101 plus CHOP (G-CHOP) to the standard R-CHOP. After a median observation of 29 months, response rates were similar for the rituximab and GA101 groups, with ORR $77.9 \%(59.5 \%$ of complete responses [CR]) and $77.4 \%$ (CR 56.7\%), respectively, as well as 3-year PFS rates (67\% and 70\%, respectively). No major unexpected events were reported, although GA101 was associated with a higher rate of neutropenia (48.3\%) and IRRs $(36.1 \%)$ than rituximab $(40.7 \%$ and $23.5 \%$, respectively). ${ }^{25}$ The final analysis with 5 -year follow-up confirmed the data of the primary analysis, demonstrating the good tolerability of G-CHOP, but without any superiority in terms of efficacy compared to R-CHOP. However, a trend toward a benefit of G-CHOP over R-CHOP was apparent in the germinal-center B-cell (GCB) subgroup, though more data are required. ${ }^{26}$ Similar data of efficacy and safety were reported in a phase II trial of Fondazione Italiana Linfomi (FIL) in elderly unfit patients treated with GA101 plus dose-reduced CHOP. ${ }^{27}$

\section{Tafasitamab}

Tafasitamab (MOR208) is an anti-CD19 humanized Ab enhanced by the modification of two amino acids in the Fc region, which increases its affinity for Fc $\gamma$ receptors. It mediates Ab-dependent cellular phagocytosis, Abdependent cellular cytotoxicity via NK cells, and direct cytotoxicity. In vitro and in vivo studies have demonstrated the efficacy of tafasitamab in lymphoproliferative diseases and in rituximab-refractory patients. ${ }^{28}$ A phase II study tested tafasitamab as a single agent at $12 \mathrm{mg} / \mathrm{kg}$ weekly in R/R NHL for 8 weeks, followed by another four doses in patients with at least stable disease (SD) and monthly until progression in partial response (PR) 


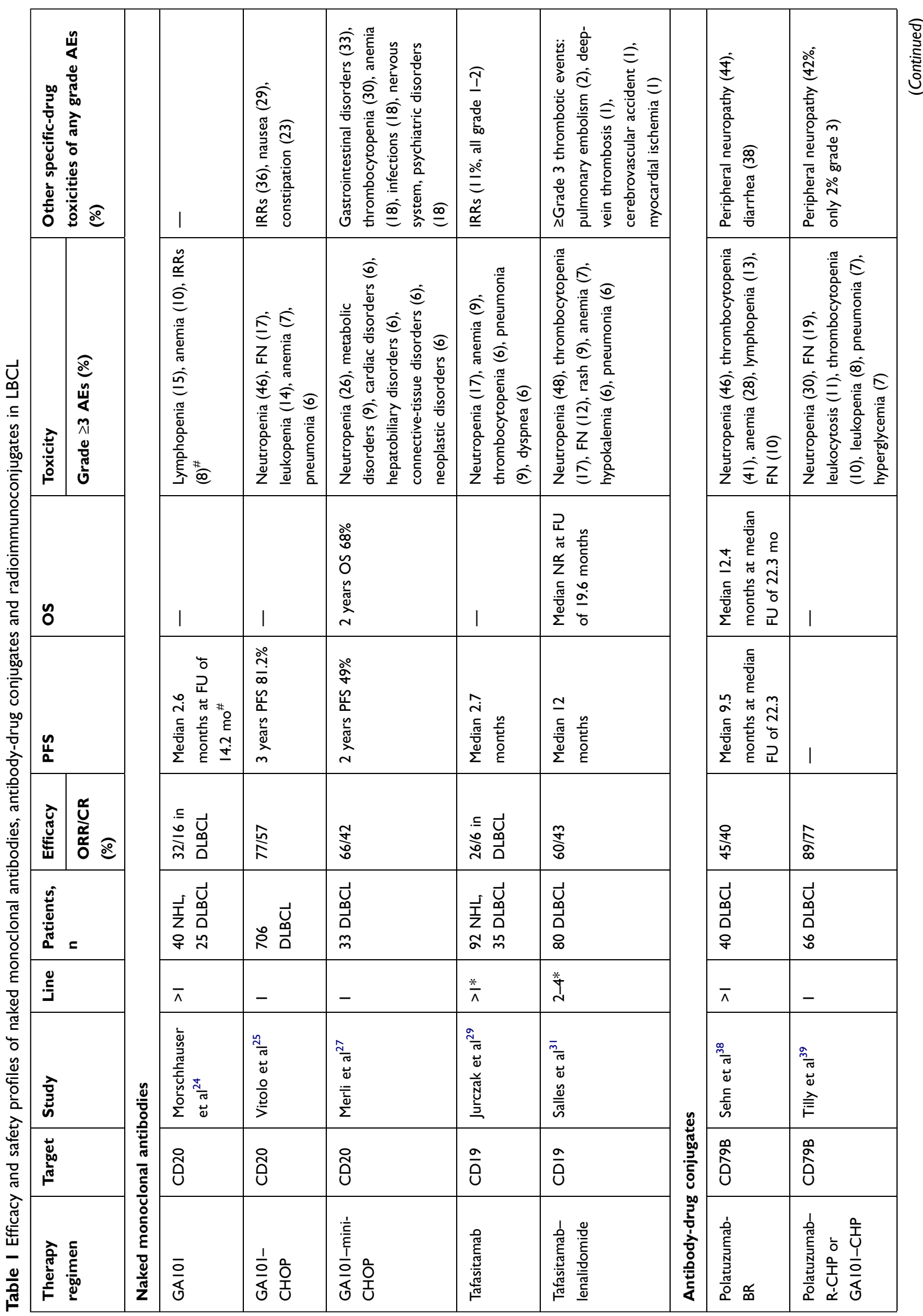




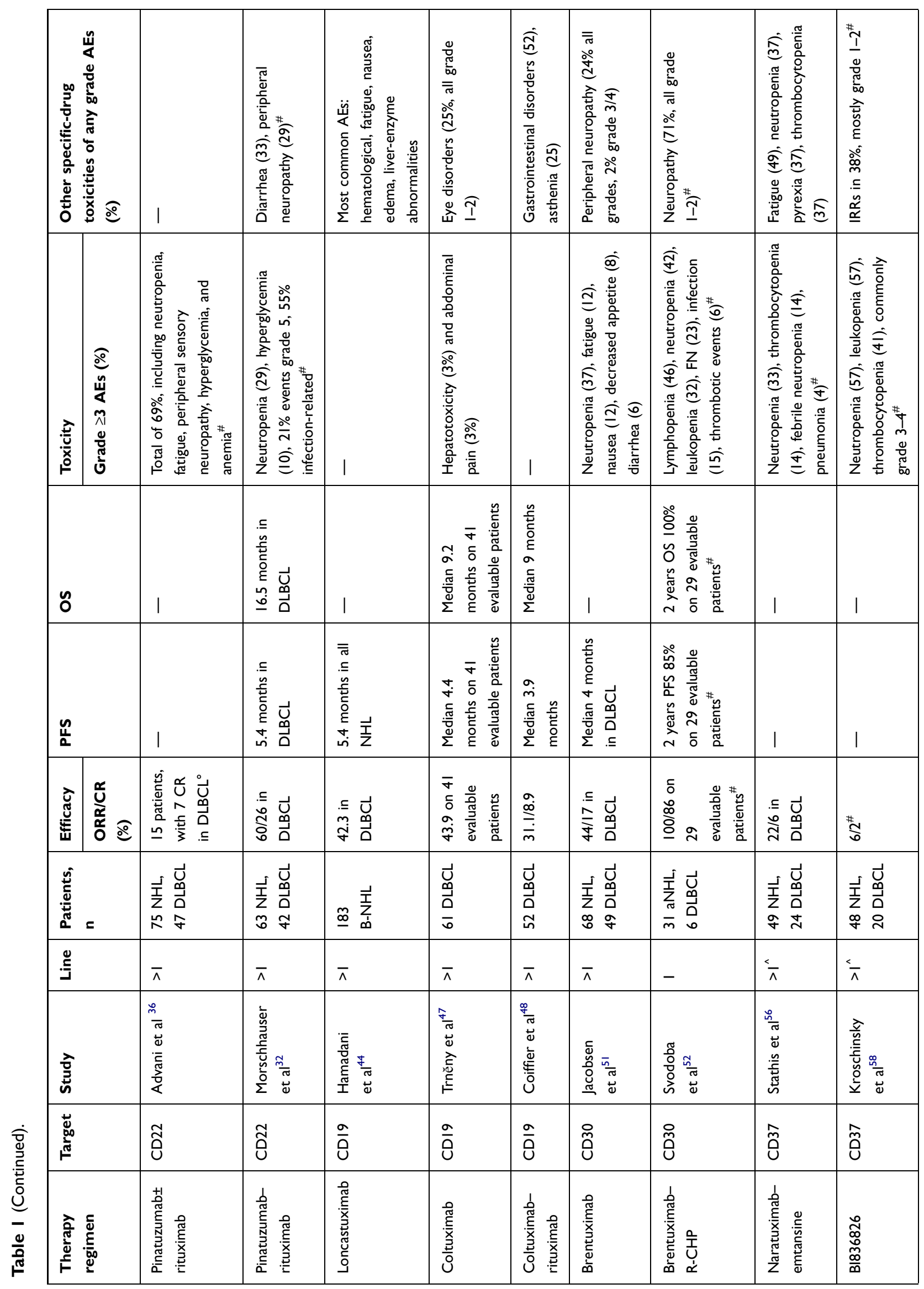




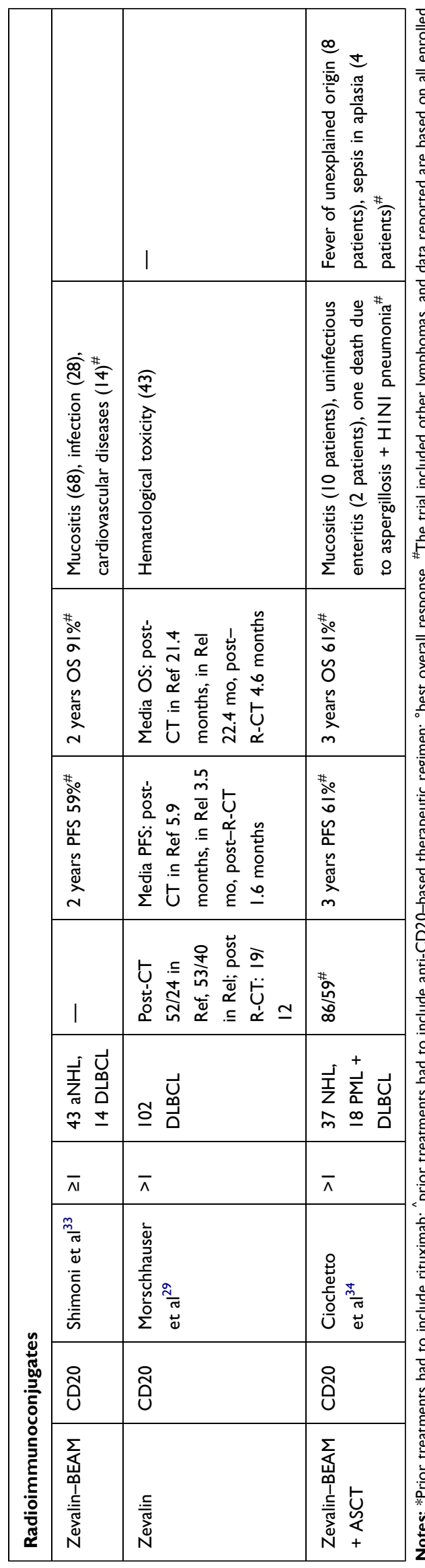

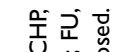
บ

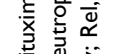

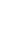

हु
andCR cases. Tafasitamab reached a $26 \%$ ORR in DLBCL, with five of nine cases with durable response (>12 months). Median duration of response (DOR) was 20.1 months, with median PFS of 2.7 months. Refractoriness to rituximab did not impact response or survival. Major AEs included IRRs and neutropenia. ${ }^{29}$ In vitro findings of enhanced NK cell-mediated cytotoxicity of tafasitamab through the addition of lenalidomide encouraged further investigations on the efficacy of this combination in a clinical setting, eg, the L-MIND trial. ${ }^{30,31}$ In this study, R/R DLBCL patients ineligible for autologous stem-cell transplantation (ASCT) received tafasita$\mathrm{mab} 12 \mathrm{mg} / \mathrm{kg}$ per day on days $1,8,15$ and 22 in cycles 1-3 and for cycle 4 twice a month for up to 12 cycles in association with lenalidomide $25 \mathrm{mg}$ /day on day 1-21 of a 28 -day cycle, followed by monthly monotherapy with tafasitamab (in SD cases) until progression. Among 80 treated patients, ORR was $60 \%$, with $43 \%$ CR and $18 \%$ PR. At a median follow-up of 17.3 months, median PFS was 12.1 months, while at a median follow-up of 19.6 months, median overall survival (OS) was not reached. The most common AE was hematological toxicity, with grade 3 or worse neutropenia in $48 \%$, thrombocytopenia in $17 \%$. and febrile neutropenia in $12 \%$, whereas other nonhematological severe events were rash in $9 \%$ and pneumonia in $9 \% .{ }^{31}$ These good results in a heavily pretreated cohort of DLBCL patients highlighted the possibility of treating these patients with a chemotherapy-free approach, and on this basis the FDA recently approved tafasitamab combined with lenalidomide $\left(\mathrm{R}^{2}\right)$ for treatment of $\mathrm{R} / \mathrm{R}$ DLBCL noncandidates for transplant. Moreover, this prompted a new phase 3 randomized study for first-line high-risk DLBCL testing the addition of tafasitamab with lenalidomide to R-CHOP vs R-CHOP alone (EudractCT 2020-002990-84).

\section{Radioimmunoconjugates}

Radioimmunoconjugates, particularly ibritumomab tiuxe$\tan$ (Zevalin), have been investigated in B-NHLs. This construct combines an antiCD20 $\mathrm{mAb}$ with the radionucleotide yttrium90, making it possible to target radiation to CD20-positive lymphoma B-cells, sparing irradiation to other normal cells (Figure 1). Zevalin has demonstrated acceptable toxicity and moderate activity as a single agent in transplant-ineligible elderly $\mathrm{R} / \mathrm{R}$ DLBCL patients. ${ }^{32}$ A phase III trial compared the pre-ASCT conditioning regimen carmustine, etoposide, cytarabine, and melphalan (BEAM) with BEAM plus Zevalin (Z-BEAM) in $R / R$ 


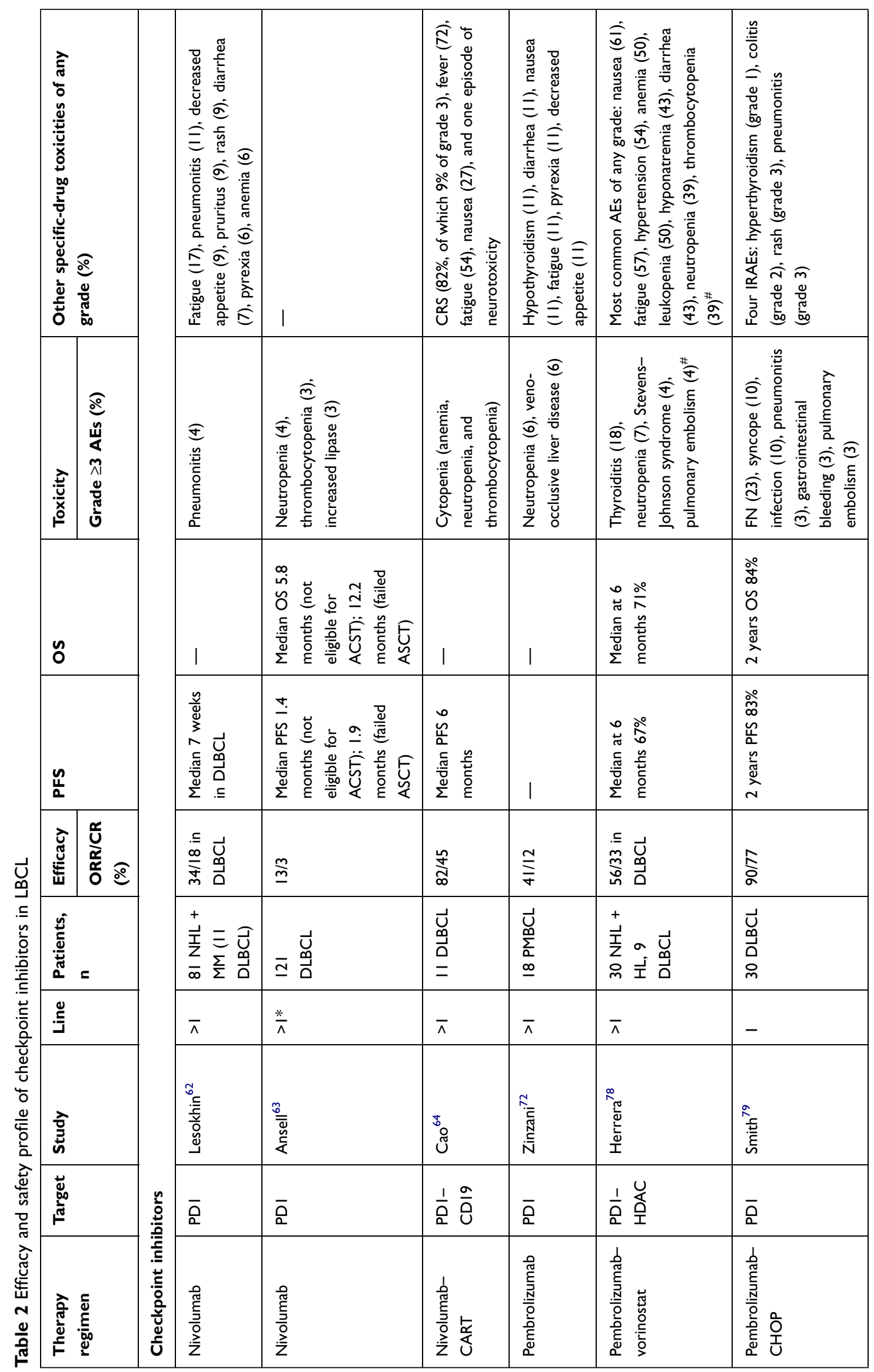




\begin{tabular}{|c|c|c|c|c|c|c|c|c|}
\hline I & & 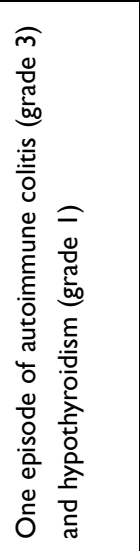 & I & 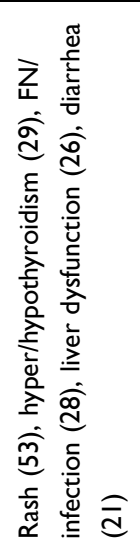 & 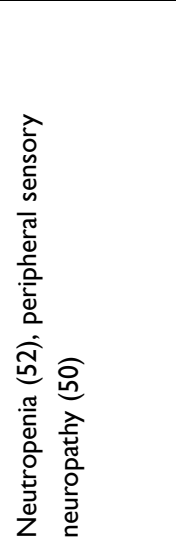 & 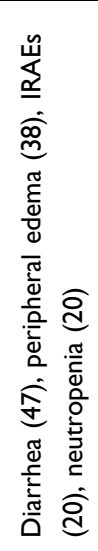 & 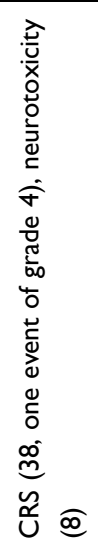 & 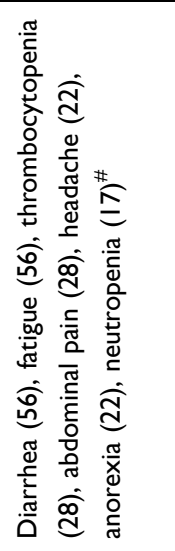 \\
\hline 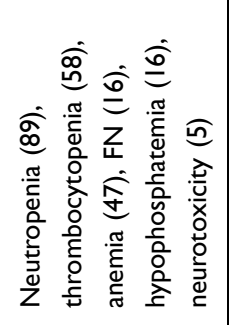 & 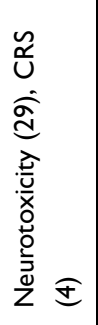 & 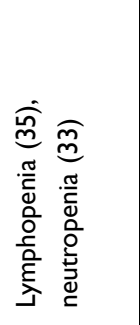 & 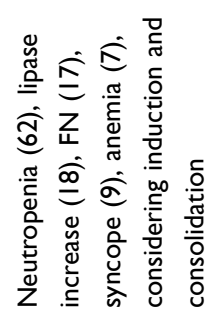 & 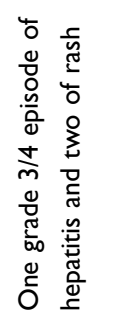 & & & & 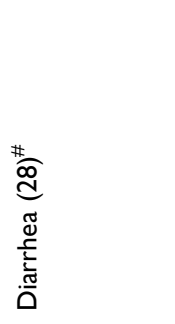 \\
\hline 1 & 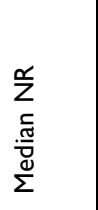 & I & 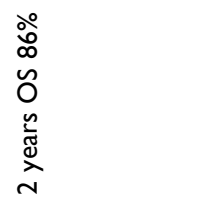 & 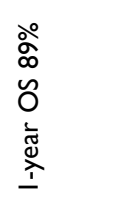 & I & 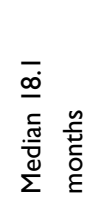 & I & I \\
\hline 1 & 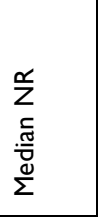 & I & 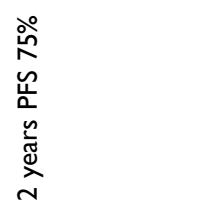 & 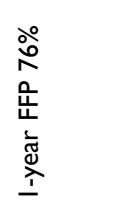 & 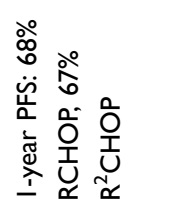 & 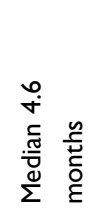 & | & 1 \\
\hline $\begin{array}{l}\text { tे } \\
\text { fे }\end{array}$ & $\frac{f}{k}$ & 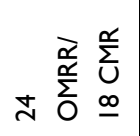 & 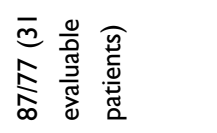 & 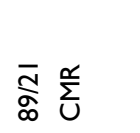 & 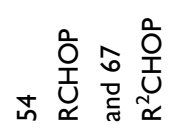 & 茎 & ₹ & 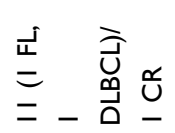 \\
\hline $\begin{array}{l}\overrightarrow{0} \\
\stackrel{0}{0} \\
\stackrel{\infty}{N}\end{array}$ & 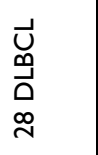 & 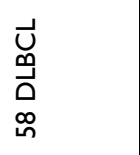 & 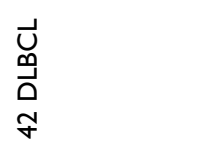 & 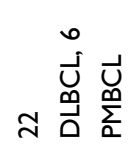 & \begin{tabular}{l}
$\overrightarrow{0}$ \\
9 \\
0 \\
\multirow{4}{f}{}
\end{tabular} & $\begin{array}{l}\vec{U} \\
\stackrel{्}{0} \\
\dot{O} \\
\dot{m}\end{array}$ & $\begin{array}{l}\stackrel{\vec{I}}{\sum_{\text {N }}} \\
\underline{\underline{n}}\end{array}$ & $\begin{array}{l}\overrightarrow{\underline{T}} \\
\underline{\underline{z}} \\
\underline{\infty}\end{array}$ \\
\hline $\bar{\Lambda}$ & $\bar{\Lambda}$ & $\bar{\Lambda}$ & - & - & - & $\bar{\Lambda}$ & $\bar{\Lambda}$ & $\bar{\Lambda}$ \\
\hline 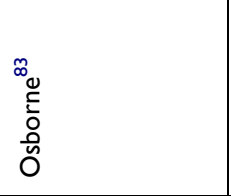 & 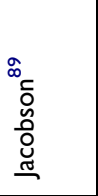 & 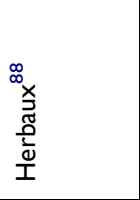 & 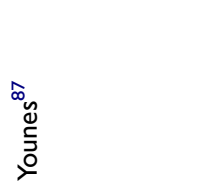 & 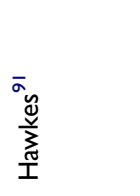 & 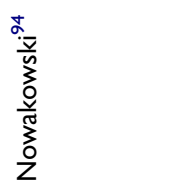 & 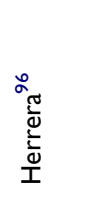 & 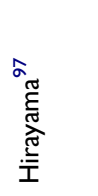 & $\frac{\stackrel{\Sigma}{\circ}}{\overline{\bar{\Phi}}}$ \\
\hline 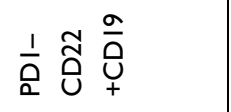 & $\overline{\bar{\alpha}}$ & 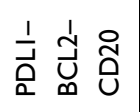 & $\overline{\bar{a}}$ & $\overline{\vec{\partial}}$ & $\overline{\vec{a}}$ & $\overline{\vec{Q}}$ & $\overline{\bar{a}}$ & $\stackrel{*}{E}$ \\
\hline 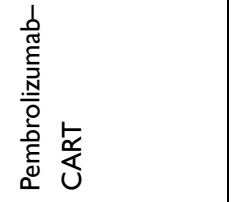 & 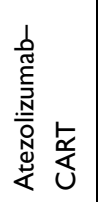 & 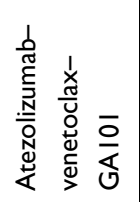 & 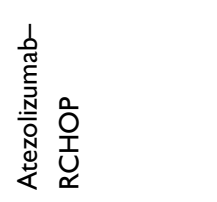 & 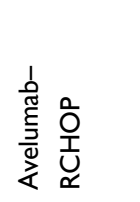 & 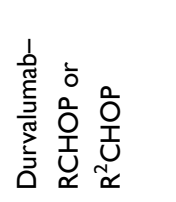 & 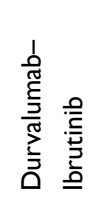 & 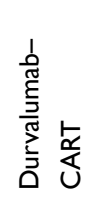 & 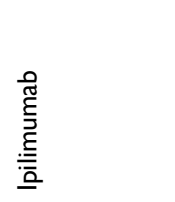 \\
\hline
\end{tabular}




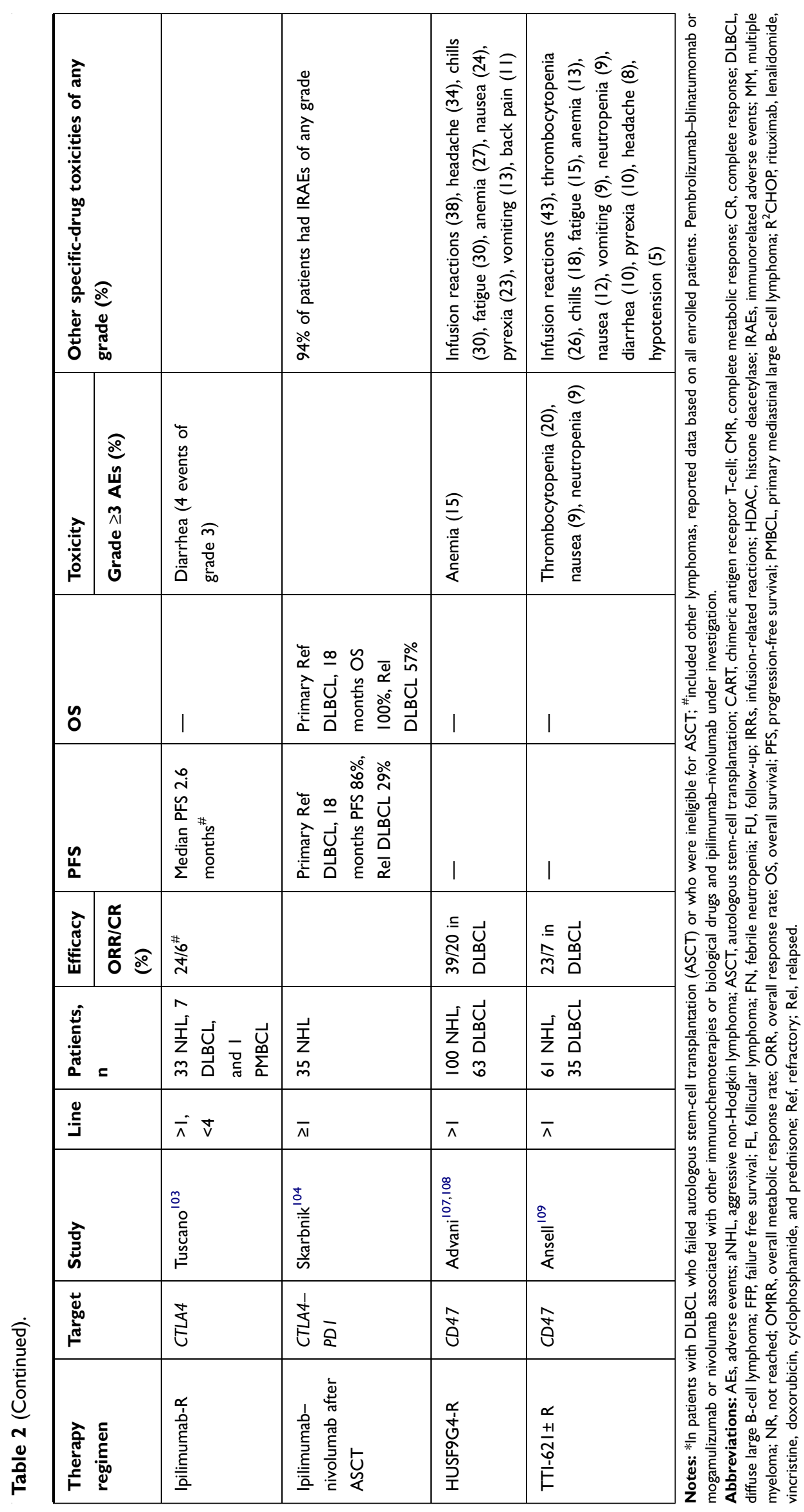




\begin{tabular}{|c|c|c|c|c|c|c|c|c|}
\hline 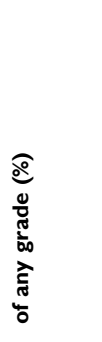 & & 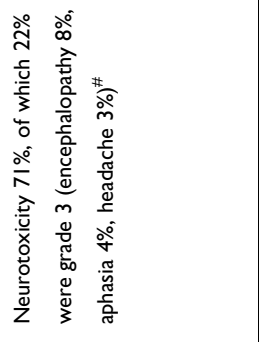 & 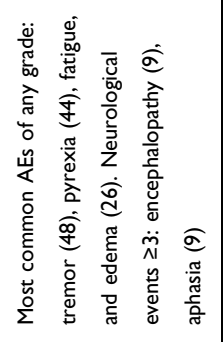 & 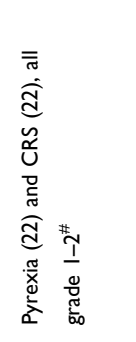 & 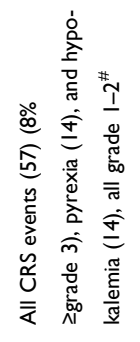 & 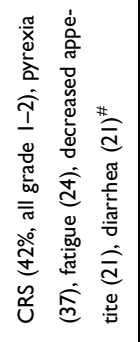 & 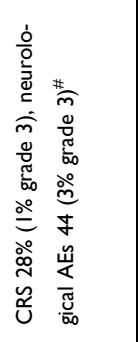 & 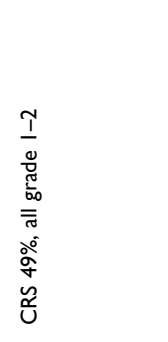 \\
\hline 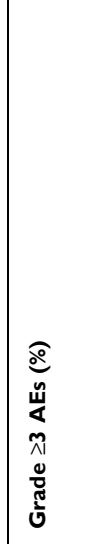 & & 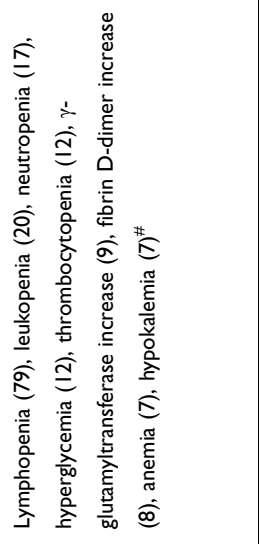 & 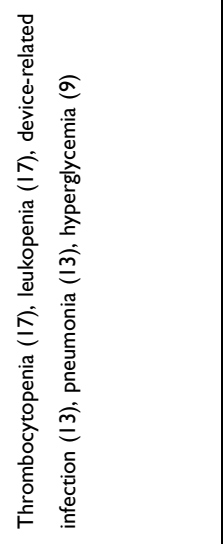 & 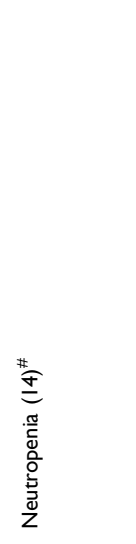 & 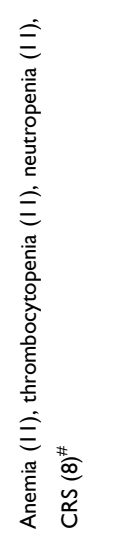 & 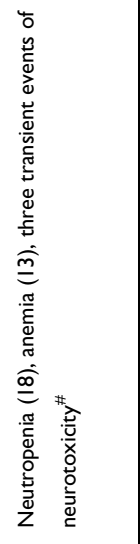 & I & 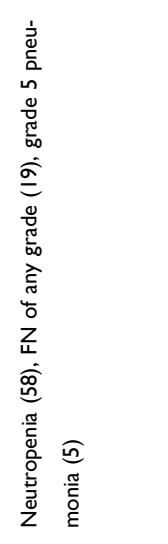 \\
\hline & & I & 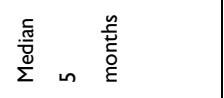 & 1 & 1 & 1 & I & 1 \\
\hline & & I & 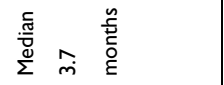 & 1 & I & I & I & | \\
\hline 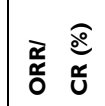 & & 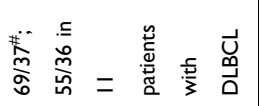 & $\stackrel{a}{\bar{y}}$ & 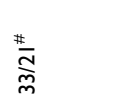 & 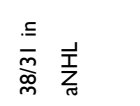 & $\stackrel{\Xi}{\cong}$ & 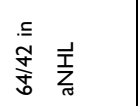 & 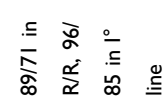 \\
\hline & & 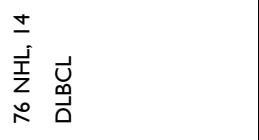 & $\begin{array}{l}\vec{U} \\
\stackrel{9}{\Delta} \\
\text { 山े }\end{array}$ & 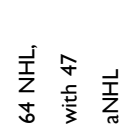 & 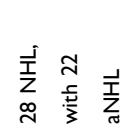 & 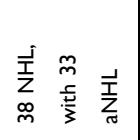 & 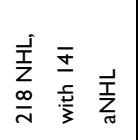 & 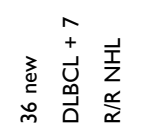 \\
\hline & & $\pi$ & $\bar{\lambda}$ & $\bar{\lambda}$ & $\bar{\lambda}$ & $\bar{\lambda}$ & $\bar{\lambda}$ & $\bar{\Lambda}$ \\
\hline & & 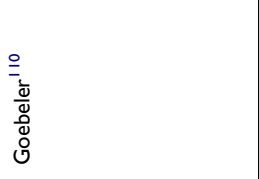 & 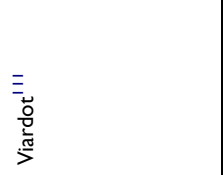 & 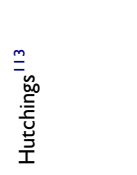 & 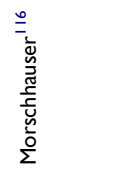 & 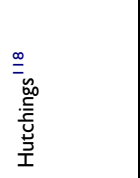 & 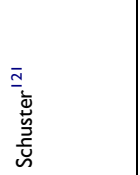 & 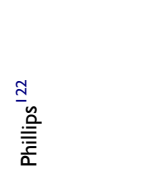 \\
\hline & & $\frac{\alpha}{0} \tilde{u}$ & $\frac{\alpha}{\bar{u}} \tilde{u}$ & ồ & ชิ̀ & 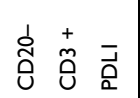 & ชิ̃ & $\stackrel{\tilde{\tilde{U}}}{\text { 0ิ }}$ \\
\hline${ }^{\bar{E}}$ & 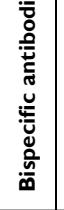 & 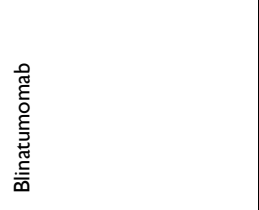 & 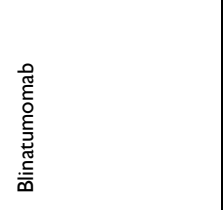 & 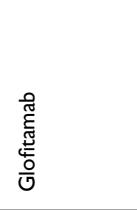 & 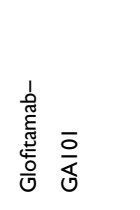 & 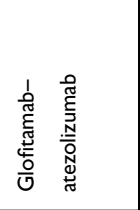 & 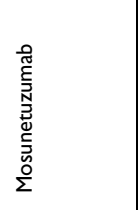 & 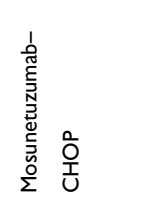 \\
\hline
\end{tabular}




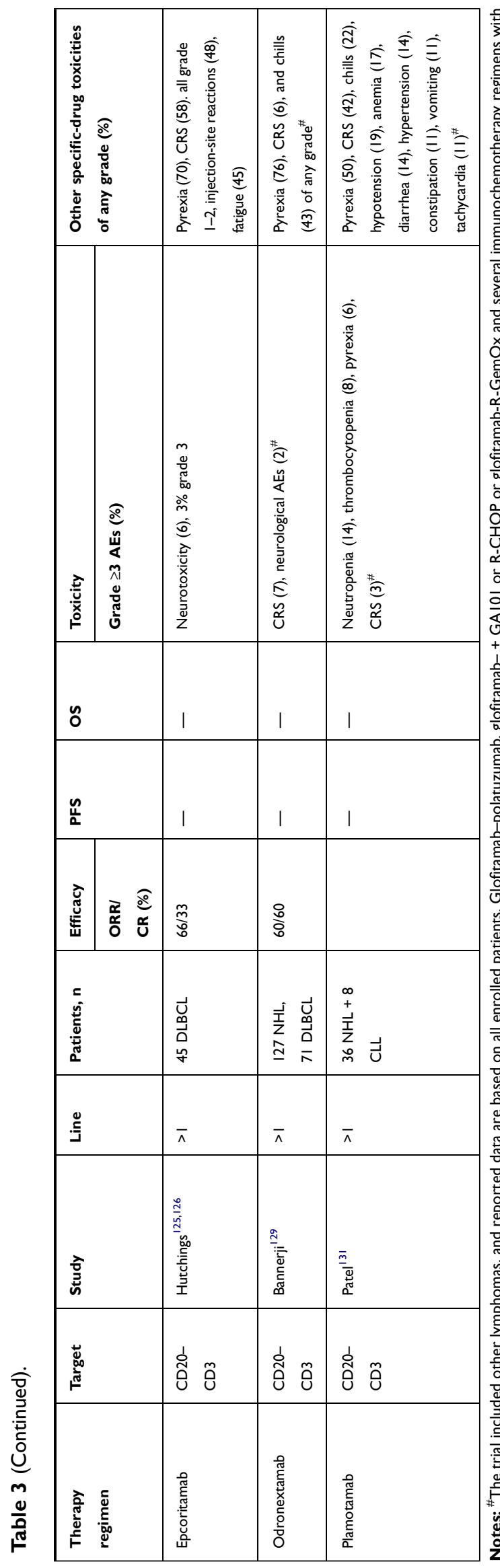

$\stackrel{\mathscr{y}}{\mathscr{\Xi}}$

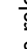
항 primary endpoint (12-year PFS) was not met, though a trend of better OS using BEAM was observed. ${ }^{33}$ Similarly, a nonsignificant trend of better PFS with the Z-BEAM-conditioning regimen compared with BEAM alone in $\mathrm{R} / \mathrm{R}$ DLBCL transplantcandidates has been reported by a single-center retrospective analysis. ${ }^{34}$ Due to a lack of clear evidence of effectiveness on DLBCL, interest in further investigations and clinical use of Zevalin for this disease is relatively sparse nowadays.

\section{Antibody-Drug Conjugates}

$\mathrm{Ab}-$ drug conjugates (ADCs) are compounds consisting of an $\mathrm{mAb}$ directed against a specific tumor-surface antigen linked with a cytotoxic drug that is delivered into malignant cells. Stable linkage between the mAb and the cytotoxic drug ensures that the drug does not detach from the $\mathrm{Ab}$, giving a highly tumor-specific effect with elevated safety and efficacy (Figure 1).

\section{Pinatuzumab Vedotin and Polatuzumab Vedotin}

Polatuzumab vedotin is an ADC comprised of an anti-CD79B $\mathrm{mAb}$ conjugated with a protease-cleavable link with monomethyl auristatin E (MMAE), a microtubule-disrupting agent. Polatuzumab vedotin demonstrates modest activity as a single agent for B-NHL, including DLBCL. ${ }^{35}$ The most frequent side effect is neutropenia, and neurotoxicity is a common treatment-emergent AE. Pinatuzumab vedotin is an anti-CD22 ADC loaded with MMAE. It has been demonstrated to be safe and active in R/R NHLs, including DLBCL, both as a single agent and combined with rituximab in a phase I study. ${ }^{36}$

More recently, a phase II, multicenter, open-label, randomized trial compared rituximab plus pinatuzumab or polatuzumab in $\mathrm{R} / \mathrm{R}$ DLBCL and $\mathrm{FL} .{ }^{37}$ For the $\mathrm{R} / \mathrm{R}$ $\mathrm{DLBCL}$ cohort, the combination rituximab + pinatuzumab demonstrated superimposable activity in respect of rituximab + polatuzumab in terms of response rates and PFS ORR $60 \%$ and $54 \%$, CR $26 \%$ and $21 \%$, and median PFS 5.4 months and 5.6 months, respectively - but in the rituximab + pinatuzumab group, grade 5 AEs were more frequent $\left(21.4 \%\right.$ vs none). ${ }^{37}$ Therefore, polatuzumab was selected for further development instead of pinatuzumab because of a better overall benefit-risk balance.

The combination polatuzumab vedotin plus bendamustine and rituximab (pola-BR) has been approved by the 


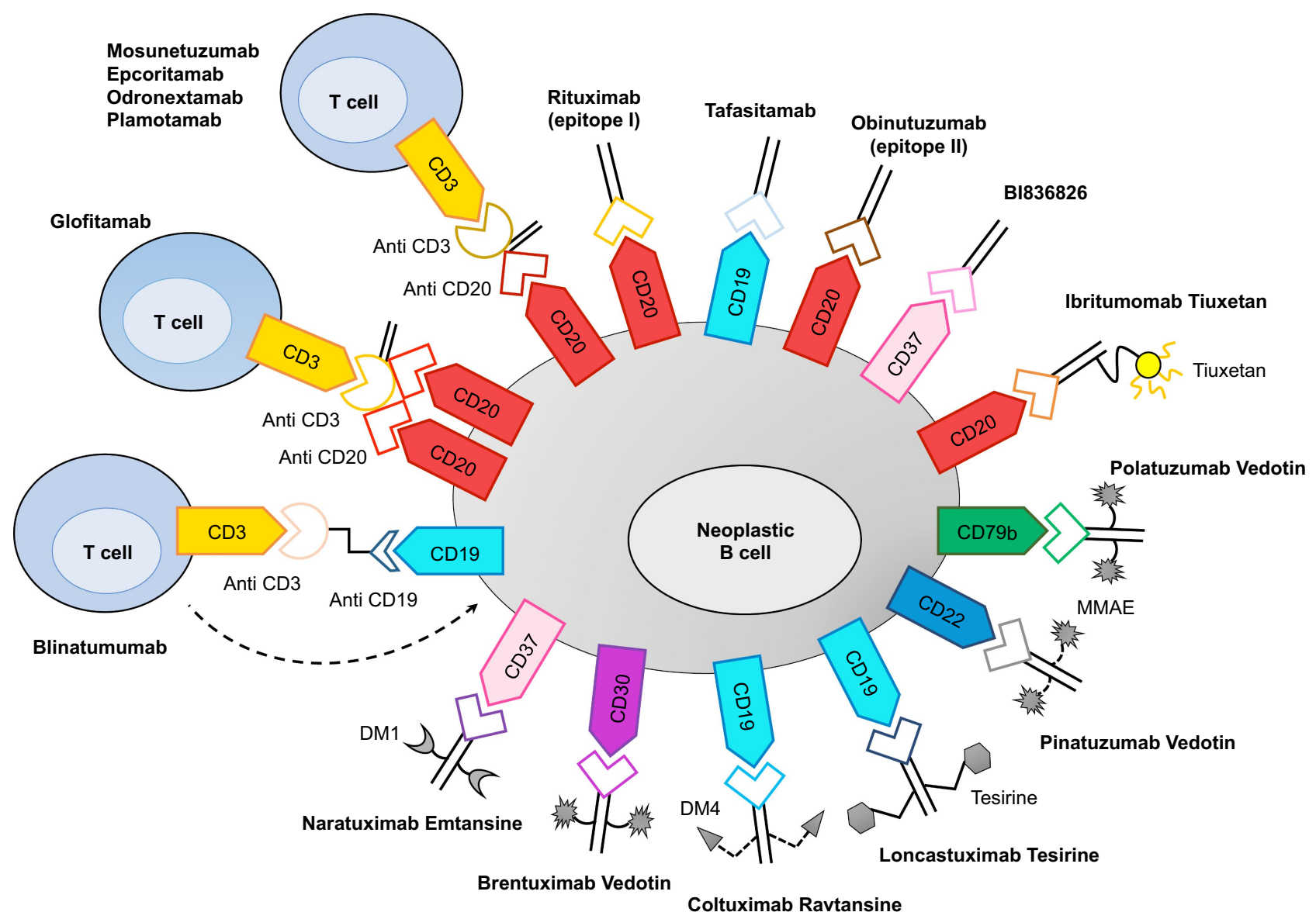

Figure I Monoclonal antibodies, including naked antibodies, antibody-drug conjugates, radioimmunoconjugates, and bispecific antibodies, are able to target Bcells on different surface antigens and with a number of cytotoxic mechanisms of action.

FDA and EMA for treatment of transplant-ineligible $\mathrm{R} / \mathrm{R}$ DLBCL, led by the results from the phase II randomized trial by Sehn et al. ${ }^{38}$ In that study, the experimental arm pola-BR compared to standard BR met the primary end point of CR improvement $>20 \%$ ( $40 \%$ and $17.3 \%$ for pola$\mathrm{BR}$ and $\mathrm{BR}$, respectively) and increased survival in terms of both PFS and OS. Polatuzumab showed a safe toxicity profile when combined with an anti-CD20 mAb (rituximab or obinutuzumab) plus cyclophosphamide, doxorubicin, and prednisone (pola-R-CHP or pola-G-CHP), omitting vincristine to avoid overlapping neurotoxicity, and a phase III trial (POLARIX) that compared the combination pola-R-CHP vs the standard R-CHOP in frontline therapy for DLBCL is ongoing. ${ }^{39,40}$ Polatuzumab vedotin is also under investigation for the treatment of $\mathrm{R} / \mathrm{R}$ transplant-eligible DLBCL in combination with ifosfamide, carboplatin, and etoposide (polaR-ICE) and in the phase III trial POLARGO, which is comparing polatuzumab plus rituximab, gemcitabine, and oxaliplatin (pola-R-GemOx) vs R-GemOx alone for R/R DLBCL. ${ }^{41,42}$

\section{Loncastuximab Tesirine}

Loncastuximab tesirine (ADCT402) is an ADC comprising a humanized anti-CD19 mAb conjugated through a cathepsin-cleavable linker to a pyrrolobenzodiazepine dimer toxin known as tesirine (SG3199). It has demonstrated high cytotoxic activity in vitro on CD19-expressing human cells. ${ }^{43}$ The final results from a phase I study testing ADCT-402 for multirefractory NHLs, including DLBCL, FL, and mantle-cell lymphoma histology have recently been published. ${ }^{44}$ ADCT showed an acceptable toxicity profile and a certain efficacy on R/R DLBCL, with ORR of $42.3 \%$ and median duration of response of 5.4 months, encouraging further investigation. ${ }^{44}$

\section{Coltuximab Ravtansine}

Coltuximab ravtansine (SAR3419) is a further compound constituted by an anti-CD19 mAb linked with a potent cytotoxic maytansinoid, DM4, via a cleavable disulfide bond. The optimal dosage of $55 \mathrm{mg} / \mathrm{m}^{2}$ weekly for four 
doses followed by four biweekly doses for R/R B-NHLs has been defined through the phase I dose-escalation study by Ribrag et al, ${ }^{45}$ with a safer toxicity profile, and especially reduction in ocular toxicities in respect of what was seen in a previous trial. ${ }^{46}$ Two phase II trials have tested SAR3419 as a single agent or combined with rituximab in $\mathrm{R} / \mathrm{R}$ DLBCL patients, with moderate efficacy (ORR $31.9 \%-43.9 \%)^{47,48}$

\section{Brentuximab Vedotin}

Brentuximab vedotin (BV) is composed of an anti-CD30 $\mathrm{mAb}$ conjugated with MMAE. Following the high efficacy demonstrated for HL, typically characterized by CD30expressing cells, $\mathrm{BV}$ has been tested on $\mathrm{R} / \mathrm{R}$ PMBCL in a phase II study, with unsatisfactory results (ORR 13\%) and early closure of the trial. ${ }^{49}$ On the other hand, promising results have been observed with the combination BV plus nivolumab for pretreated PMBCL patients, with 70\% ORR, $43 \%$ CR, and median PFS and OS not reached after a median follow-up of 11.1 months. ${ }^{50} \mathrm{BV}$ was also tested for CD30-positive R/R DLBCL patients, showing moderate activity (ORR 44\%, CR 17\%), irrespective of the grade of expression of $\mathrm{CD} 30$, and a safe profile. ${ }^{51}$ In the same phase I/II trial, the combination with rituximab was also evaluated, with no additional benefit. ${ }^{51}$ Moreover, in a phase I/II trial, BV has been combined with R-CHP (BV+R-CHP) as frontline treatment for CD30-positive B-NHL, including 22 PMBCL, 5 DLBCL, and two gray-zone lymphomas, with promising toxicity profile and efficacy (ORR $100 \%$, CR $86 \%$ ). ${ }^{52}$ A phase III trial investigating the efficacy of rituximab and lenalidomide with or without $\mathrm{BV}$ for $\mathrm{R} / \mathrm{R}$ transplant-ineligible patients is ongoing. ${ }^{53}$

\section{Anti-CD37 Compounds}

CD37 is a tetraspanin expressed on both healthy and malignant B-cell surfaces that plays a crucial role in cell survival and response of immune system. ${ }^{54}$ Lower expression of CD37 in DLBCL cells has been associated with worse survival after immunochemotherapy, suggesting that CD37 may optimize rituximab activity. ${ }^{55}$ These data encouraged the development of several anti-CD37 drugs for hematological neoplasms. Naratuximab emtansine, an anti-CD37 mAb conjugated with the potent antimitotic maytansine-derived microtubule disruptor (DM1), has been tested on 49 patients with R/R NHLs, resulting in $13 \%$ ORR (one CR and four PR), and a phase II trial in combination with rituximab on $\mathrm{R} / \mathrm{R}$ NHL including
DLBCL is ongoing. ${ }^{56,57} \mathrm{BI} 836826$, an anti-CD37 chimeric $\mathrm{Ab}$, was tested on NHL in a phase I trial and demonstrated modest results, with response in three of 48 patients (one $\mathrm{CR}$ ), despite a safe profile, with major AEs represented by hematological toxicities and manageable IRRs. ${ }^{58}$ Other mAbs targeting CD37 include otlertuzumab, which has been studied in combination with bendamustine for CLL, AGS67E, an ADC conjugated with MMAE tested in AML and lymphoproliferative diseases, and betalutin, a murine radioimmunoconjugate, linked with the $\beta$-emitting isotope lutetium177, studied in preclinical models of NHL in association with rituximab, showing an improvement in anti-CD20 activity. ${ }^{59-61}$

\section{Checkpoint Inhibitors}

The promising therapeutic effects of checkpoint inhibitors have attracted great interest in the past decade and have undoubtedly revolutionized cancer treatment. Several mAbs capable of targeting cellular immunocheckpoints have been developed and investigated in the treatment of patients with LBCL. Checkpoint inhibitors work by disrupting the interaction between inhibitory receptors and their ligands and thus activating antitumor immunity. The compounds most actively studied for the treatment of LBCL are molecules that target PD1/PDL1, CTLA4), and CD47 (Figure 2, Table 2).

\section{Anti-PDI/PDLI Compounds}

A member of the CD28 family, is a receptor expressed on the surface of $\mathrm{T}$ cells that is able to regulate its activation by interacting with two ligands (PDL1 and PDL2). PDL1 is expressed in a number of different cell types, as well as tumor cells. The interaction of PD1 with PDL1 or PDL2 results in the inhibition of $\mathrm{T}$ cell-receptor signaling and downregulates T-cell proliferation. Cancer cells exploit PD1 signaling to escape $\mathrm{T}$ cell-mediated eradication. Highly selective mAbs directed to PD1 (eg, pembrolizumab and nivolumab) or PDL1 (eg, atezolizumab, avelumab, and durvalumab) can disrupt receptor-antigen interaction and reverse T-cell inactivation, restoring the immune-system antitumor response.

\section{Nivolumab}

Nivolumab is another anti-PD1 mAb, nowadays approved for the treatment of $\mathrm{HL}$ patients that resulted $\mathrm{R} / \mathrm{R}$ to ASCT. In the setting of R/R DLBCL, nivolumab as monotherapy was tested on eleven patients in a phase I trial with modest results. ORR was 36\% (CR 18\%) andmedian PFS 

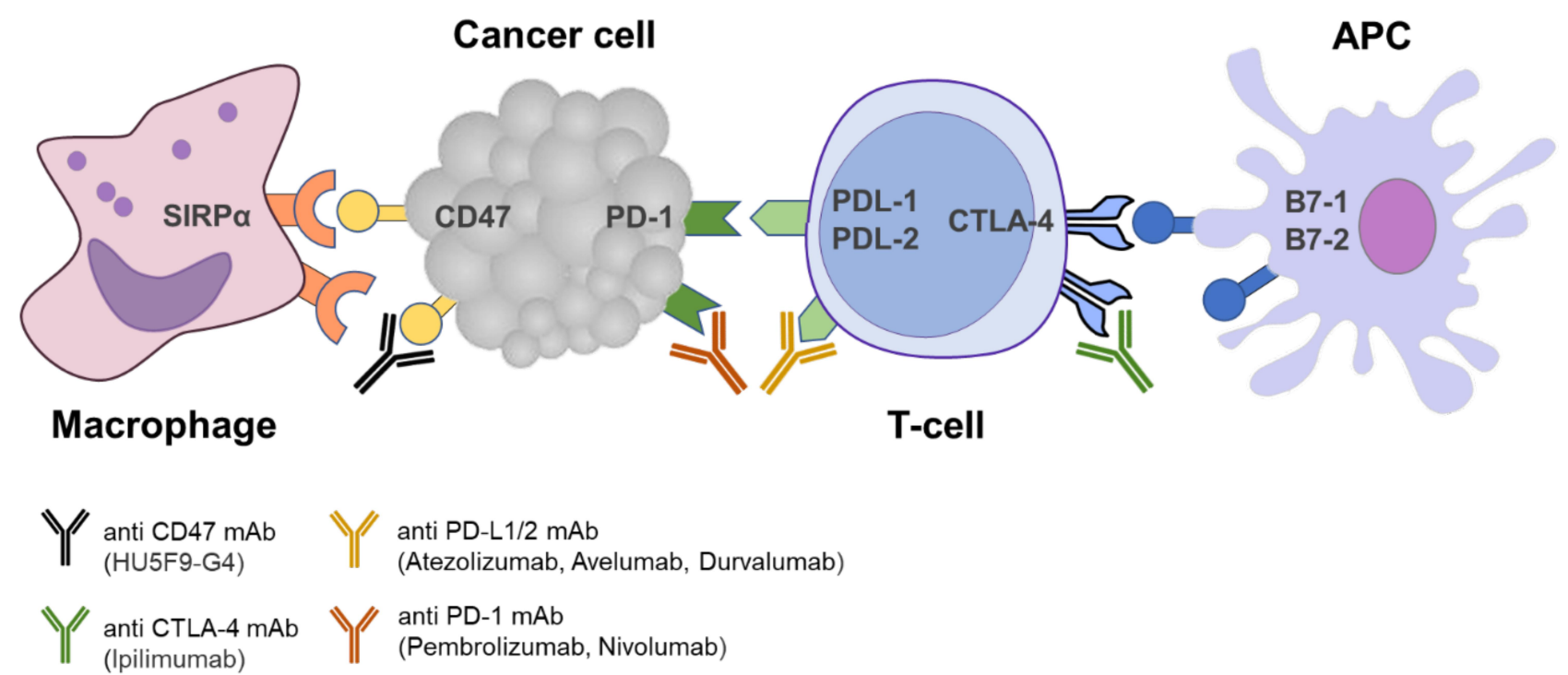

Figure 2 Checkpoint inhibitors (anti-CD47, anti-PDLI/2, antiCTLA4, and anti-PDI) disrupt interaction between inhibitory receptors and their ligands and thus activate antitumor immunity.

7 weeks: one of four patients in this group has had an ongoing response, and two continue to be followed. ${ }^{62}$ Nivolumab has been reevaluated in terms of efficacy and safety in patients ineligible for ASCT. However, the results of a phase II study showed a low incidence of objective response (benefiting only a small subset of patients: ORR $10 \%$ among subjects treated following relapse following ASCT, and only 3\% in ASCT-ineligible patients) despite a favourable safety profile. ${ }^{63}$

Nivolumab in combination with other anticancer therapies has been investigated in $\mathrm{R} / \mathrm{R}$ DLBCL. A single-center study evaluated safety and efficacy of the combination anti-CD19 CART plus nivolumab for R/R B-NHL, showing manageable toxicity and opening up opportunities for further clinical trials. In this study oneleven R/R LBCL subjects, ORR and CR were $81.8 \%$ and $45.4 \%$, respectively. With median follow-up of 6 months,median PFS was 6 months. ${ }^{64}$ A number of further phase I/II studies are currently being carried out to investigate the therapeutic value of nivolumab with other antilymphoma treatments, such as rituximab and chemotherapy regimens, ipilimumab, varlilumab (anti-CD27 mAb), lenalidomide, rituximab + lenalidomide $\left(\mathrm{R}^{2}\right)$, and epacadostat (an IDO1-selective inhibitor) in LBCL patients. $^{65-71}$

\section{Pembrolizumab}

Pembrolizumab has been the first humanized anti-PD1 mAb to receive accelerated FDA clearance for the treatment of R/R PMBCL. This approval was based on results of the phase IB
KEYNOTE-013 and phase II KEYNOTE-170 trials, which demonstrated a high response rate, durable activity, and favorable safety profile in a subset of PMBCL patients. $^{72,73}$ A number of studies are currently evaluating the efficacy of pembrolizumab in DLBCL patients, either as single therapy or in combination with other drugs. ${ }^{7-77}$ Of note, PD1 ligands appear to be poorly expressed in DLBCL, with $27 \%$ of tumor specimens being PDL1-positive onimmunohistochemistry, leading to low $\mathrm{CR}$ rates or no response to anti-PD1. However, the presence of $P D L 1$ gene alterations has been associated with objective response to pembrolizumab in $\mathrm{R} / \mathrm{R}$ DLBCL patients. An ongoing phase II study has been designed to fully evaluate the possibility of using $P D L 1$ genetic alterations in $\mathrm{R} / \mathrm{R}$ DLBCL to predict response to PD1 blockade.

The efficacy of pembrolizumab as monotherapy in DLBCL is still a matter of scientific debate. More success seems to have been achieved using pembrolizumab combined with other therapies. A combination of pembrolizumab and the oral histone-deacetylase inhibitor vorinostat has been tested by Herrera et al, ${ }^{78}$ showing preliminary promising results on nine $\mathrm{R} / \mathrm{R}$ transplant-ineligible DLBCL patients (ORR 56\%, CR 33\%).

Combination of pembrolizumab and R-CHOP in untreated patients with DLBCL has also been evaluated, ${ }^{79}$ demonstrating a safe toxicity profile. Among 30 patients treated, ORR and CR were $90 \%$ and $77 \%$, respectively. After a median follow-up of 25.5 months, 2-year PFS of $83 \%$ was reached. 
The use of pembrolizumab after anti-CD19 chimeric antigen-receptor T-cell (CAR-T) therapy has been investigated. The PD1 blockade demonstrated interesting activity in this setting, enhancing the efficacy of CAR- $T$ in $R / R$ LBCL. $^{80}$ Based on these findings, multiple clinical trials looking at different aspects of the synergy between pembrolizumab and CAR-T have been initiated. ${ }^{81,82}$ Promising results came from the phase I/II ALEXANDER trial, where combination of the bispecific anti-CD19/22 CAR$\mathrm{T}$ (AUTO3) and pembrolizumab induced high response rates without causing some of the key severe side effects (ie, cytokine-release syndrome [CRS] and neurotoxicity). Across four cohorts treated with different doses of AUTO3 alone or in combination with pembrolizumab, ORR was $68 \%$ and CR $54 \%{ }^{83}$ Contrarily, the consolidative use of pembrolizumab after ASCT for patients with R/R DLBCL has been investigated through a phase II multicenter study, but with no improvement in terms of PFS. ${ }^{84}$ Other studies are currently looking into the combination of pembrolizumab with other drugs, such as the CD3xCD19 bispecific $\mathrm{mAb}$ blinatumomab and the anti-CCR4 mogamulizumab for R/R DLBCL. ${ }^{85,86}$

\section{Atezolizumab}

Atezolizumab is a fully humanized $\operatorname{IgG}_{1} \mathrm{mAb}$ targeting PDL1. Atezolizumab has been tested in combination with R-CHOP followed by consolidation with single-agent atezolizumab in previously untreated DLBCL patients. Preliminary data from this open-label phase I/II study are promising: among 40 patients who received at least one dose of atezolizumab, ORR of $87.5 \%$ and $77.5 \%$ CR have been obtained, with 2-year PFS and OS of $74.9 \%$ and $86.4 \%$, respectively. However, nonnegligible toxicity has been observed, with AEs causing a high number of discontinuations ( $36 \%$ of patients), even if they appeared to be overall manageable and reversible. ${ }^{87}$

More recently, the combination atezolizumab plus obinutuzumab and venetoclax has been tested through a multicenter phase II trial in DLBCL patients who had failed at least one line of therapy. Preliminary analysis demonstrated durable response (ORR 23.6\%) with a manageable safety profile. ${ }^{88}$ The safety and efficacy of atezolizumab in combination with the anti-CD19 CAR-T cell axicabtagene ciloleucel (axi-cel) for $\mathrm{R} / \mathrm{R}$ LBCL is under investigation in a phase $\mathrm{I} / \mathrm{II}$ trial. The interim analysis demonstrated that PDL1 blockade with atezolizumab after axi-cel was well tolerated, and the study did not reveal increased incidence of AEs. However, efficacy and
CAR-T cell levels reported in the study were comparable to those of patients treated with axi-cel alone. ${ }^{89}$

\section{Avelumab}

Similarly to atezolizumab, avelumab acts by targeting the PD1 pathway at the ligand level. In R/R DLBCL, a twocomponent phase IB/III study tested avelumab in combination with rituximab, utomilumab (a 41BB agonist) and chemotherapy drugs (ie, azacitidine, bendamustine, gemcitabine, and oxaliplatin). However, the phase III part of the study was never conducted, due to early closure of phase IB enrolment. ${ }^{90}$ Another phase II multicenter singlearm trial is investigating the feasibility of adding induction and maintenance with avelumab to standard R-CHOP therapy in patients with stage II-IV DLBCL. At the time of the interim analysis, the trial had enrolled 28 patients and reported ORR and CR after R-CHOP of $89 \%$. The ORR to two cycles of induction avelumab + rituximab (AvR) was 60\%. Six patients (21\%) progressed during AvR induction (with one completing only one AvR cycle), and all subsequently responded to R-CHOP. With a median follow-up of 16 months, 1-year failure-free survival was $76 \%$ and OS $89 \% .{ }^{91}$ The side effects and optimal dosing of avelumab, utomilumab, rituximab, ibrutinib, and combination chemotherapy are also being evaluated in a phase I clinical trial for R/R aggressive B-NHL (aNHL), including DLBCL. ${ }^{92}$

\section{Durvalumab}

The anti-PDL1 molecule durvalumab has been studied for the treatment of DLBCL as both a single-agent and in the context of combined immunochemotherapy regimens. FUSION NHL-001 is a phase I/II study assessing the safety and efficacy of durvalumab as monotherapy or in combination. Among a total of 38 patients with $\mathrm{R} / \mathrm{R}$ DLBCL enrolled, aside acceptable toxicity, the therapy had limited benefit. ${ }^{93}$

The safety and efficacy of durvalumab plus R-CHOP or $\mathrm{R}^{2} \mathrm{CHOP}$ in untreated high/high-intermediate risk DLBCL patients were assessed in a phase II open-label study. Patients received durvalumab $+\mathrm{R}-\mathrm{CHOP}$ if considered GCB-DLBCL (arm A) or $\mathrm{R}^{2} \mathrm{CHOP}$ if activated B-cellDLBCL (arm B), based on cell of origin defined by gene-expression profiling. $\mathrm{CR}$ at end of induction was $54 \%$ in arm $A$ and $67 \%$ in arm B out of a total of 46 patients treated. Fatigue, neutropenia, peripheral sensory neuropathy, and nausea were the most common AEs. ${ }^{94}$ 
A phase IB study evaluated the combination durvalumab plus tremelimumab (an anti-CTLA4 mAb) or danvatirsen (an antisense oligonucleotide anti-STAT3) for R/R DLBCL. These regimens were generally well tolerated, but had limited therapeutic effectiveness (ORR 6.3\%). ${ }^{95}$

In another phase IB/II multicenter open-label study that included GCB and non-GCB DLBCL subjects, the combination ibrutinib plus durvalumab had modest efficacy. ORR was $13 \%$ and $38 \%$ in GCB and non-GCB DLBCL patients, respectively, with AEs reported in $20 \%$. It was concluded that ibrutinib and durvalumab had similar activity to ibrutinib alone, but with added toxicity typical of PDL1 blockade. ${ }^{96}$ Durvalumab has also been used in combination with the anti-CD19 CAR-T JCAR014 in a phase I dose-finding study, showing a good safety profile. Of note, CRs were observed at both initial restaging after JCAR014 infusion and in patients continuing durvalumab therapy after initially failing to achieve $\mathrm{CR} .{ }^{97}$

Other clinical trials have been initiated to assess the activity of durvalumab after ASCT or combined with other mAbs or lenalidomide, but these have either been terminated or withdrawn for different reasons. ${ }^{98-100}$

\section{CTLA4 Blockade}

CTLA4, also known as CD152, is a receptor that belongs to the Ig superfamily and is expressed on activated $\mathrm{CD} 4^{+}$ and $\mathrm{CD}^{+}$lymphocytes. Activation of $\mathrm{T}$ lymphocytes results in upregulation of CTLA4 and its interaction with the B7 ligand, which is expressed on antigen-presenting cells and transduces an inhibitory signal to lymphocytes, thus maintaining immunoresponse homeostasis. ${ }^{101}$

Ipilimumab is a humanized $\mathrm{IgG}_{1} \kappa \mathrm{mAb}$ directed against the CTLA4 immunocheckpoint.

The first phaseI/II trial evaluating ipilimumab in a relapse setting was conducted in 2004 on 18 B-NHL patients. Only two were able to be evaluated, both showing clinical responses (CR of $31+$ months for one DLBCL patient and PR up to 19 months for an FL patient). ${ }^{102}$ The combination of ipilimumab and rituximab has been assessed through a phase I trial on R/R B-NHL, including DLBCL $(n=7)$. Manageable toxicity and limited efficacy were observed for the entire cohort (ORR 24\% and CR $6 \%$, with only FL patients showing promising responses. ${ }^{103} \mathrm{~A}$ phase IB trial has been conducted investigating combined checkpoint inhibition with ipilimumab and nivolumab after ASCT in hematological malignancies, including DLBCL. PFS and OS at 18 months post-ASCT were $85.7 \%$ and $100 \%$, respectively, for primary refractory
DLBCL and $28.6 \%$ and $57.1 \%$ for relapsed DLBCL. A manageable safety profile was also seen, indicating the combination's potential to improve outcomes following ASCT and response in patients with high-risk disease. ${ }^{104}$ Another open-label phase I/II multicenter trial evaluating dual checkpoint-blocking Ab therapy with ipilimumab and nivolumab for $\mathrm{R} / \mathrm{R}$ transplant-ineligible $\mathrm{DLBCL}$ is ongoing. ${ }^{67}$

\section{Anti-CD47 Compounds}

CD47 is a membrane receptor belonging to the Ig superfamily and involved in a number of physiological processes, including cellular migration and $\mathrm{T}$ lymphocyte/ dendritic cell activation. In addition, it can inhibit phagocytosis by interacting with expressed on phagocytes. ${ }^{105} \mathrm{By}$ doing so, CD47 mediates the "do not eat me" signal, preventing the phagocytes from eliminating damaged and senescent cells. CD47 is ubiquitously expressed on normal cells; however, it has been demonstrated that malignant hematological cells, including DLBCL ones, overexpress this receptor, leading to tumor immunoevasion by preventing phagocytosis. ${ }^{106}$ As such, CD47 blockade inhibits the CD47-SIRP $\alpha$ signaling pathway and prevents tumor progression by promoting phagocytosis of tumor cells.

Hu5F9-G4 (magrolimab, 5F9) is a humanized $\mathrm{IgG}_{4} \mathrm{Ab}$ macrophage immunocheckpoint inhibitor blocking CD47. Combination of 5F9 with rituximab was investigated for the first time in a phase IB/II study on 110 multirefractory B-NHL patients (63 DLBCL, $35 \mathrm{FL}$, two marginal-zone lymphomas). $5 \mathrm{~F} 9$ plus rituximab was well tolerated, with most AEs being grade 1-2. Among 46 evaluable DLBCL patients, ORR was $39 \%$ and CR $20 \% .{ }^{107,108}$ Based on this encouraging safety/efficacy profile, a phase IB/II study investigating a combination of 5F9-rituximab plus GemOx for $\mathrm{R} / \mathrm{R}$ indolent lymphomas and DLBCL has been designed. ${ }^{108}$

TTI-621 is a novel immunocheckpoint inhibitor that has received attention for the treatment of DLBCL. It is a recombinant fusion protein of the CD47-binding domain of SIRP $\alpha$ linked to the $\mathrm{Fc}$ region of human $\mathrm{IgG}_{1}$ that (similarly to 5F9) enhances phagocytosis and antitumor activity. A phase I trial is evaluating the safety and activity of TTI-621 as monotherapy and in combination with rituximab in hematological malignancies (including DLBCL), with encouraging results. AEs have included IRRs, thrombocytopenia, chills, and fatigue. In DLBCL subjects, ORR was $29 \%$ with TTI- 621 monotherapy and $21 \%$ with TTI621 plus rituximab. ${ }^{109}$ 


\section{Bispecific Antibodies}

The term "bispecific Ab" (bsAb) defines a family of compounds designed to recognize two specific antigens or epitopes. The therapeutic activity of these molecules is led by the redirection of autologous $\mathrm{T}$ cells against tumor cells. bsAbs come in many formats, ranging from small molecules constituted by two antigen-binding fragments to entireIgG-like compounds enriched by an additional domain, engineered to target different antigens or epitopes on tumor cells. Several bsAbs are in development for treatment of B-NHLs and show promising activity, even in heavily pretreated patients (Figure 1, Table 3).

\section{Blinatumomab}

Blinatumomab is one of the first bsAbs tested on hematological malignancies, and is approved for treatment of $R / R$ and MRD-positive B-cell acute lymphoblastic leukemia. This construct consists of two single-chain variable fragments that bind CD3 on T cells and CD19 on B cells, leading cytotoxic T-cell activity on B-lymphoma and normal cells. It has been tested on $76 \mathrm{R} / \mathrm{R}$ B-NHLs (including 14 DLBCL) in a phase I dose-escalation trial, demonstrating good tolerability and promising antilymphoma activity (ORR 69\%). ${ }^{110}$ In a phase II trial on R/R DLBCL, blinatumomab monotherapy showed moderate effectiveness, with ORR $43 \%$ and $19 \% \mathrm{CR}$, and main grade 3 toxicities (neurological events, including aphasia [9\%] and encephalopathy [9\%]), mostly resolved. ${ }^{111} \mathrm{~A}$ phase II/III trial investigating the efficacy of blinatumomab in DLBCL not achieving complete metabolic response after a platinum-based salvage regimen is ongoing. ${ }^{112}$

\section{Glofitamab}

Glofitamab is a bsAb with a 2:1 molecular structure consisting of two CD20-binding fragments and a single CD3binding fragment, which can potentially increase tumorantigen avidity. ${ }^{113}$ Glofitamab has been tested in a phase I trial in R/R B-NHLs, preceded by a $1,000 \mathrm{mg}$ dose of obinutuzumab administered 7 days before the start of treatment, in order to debulk peripheral B cells and reduce systemic cytokine release. ${ }^{113,114}$ Preliminary data of the first-in-human phase I trial on $64 \mathrm{R} / \mathrm{R}$ B-NHLs (47 aNHLs, including DLBCL and PMBCL) showed promising antitumor activity and manageable safety: among the subgroup of aNHLs treated with $300 \mu \mathrm{g}$ or above, 33\% ORR and $21 \%$ CR were observed, with the most frequent toxicity being transient CRS (22\%, all grade 1 or 2$){ }^{113}$
Durable response and good tolerability have been confirmed in the updated analysis of the expansion cohort. ${ }^{115}$ The promising results of single-agent glofitamab prompted further investigations with several treatment combinations. Interesting antilymphoma activity and an adequate safety profile have been reported from the preliminary data of the combination of glofitamab and obinutuzumab in a phase IB trial. ${ }^{116}$ Another phase IB study combining glofitamab with atezolizumab or polatuzumab vedotin for $R / R$ B-NHLs is recruiting. ${ }^{117,118}$ Glofitamab plus immunochemotherapy (R-CHOP or G-CHOP) for R/R B-NHL and untreated DLBCL is under investigation, as well as a comparison of R-GemOx vs glofitamab + R-GemOx in a randomized phase III trial dedicated to $\mathrm{R} / \mathrm{R}$ transplantineligible or refractory DLBCL. ${ }^{19,120}$

\section{Mosunetuzumab}

Mosunetuzumab is a full-length fully humanized $\operatorname{IgG}_{1}$ $\mathrm{CD} 3 \mathrm{xCD} 20 \mathrm{bsAb}$. It has been investigated by Schuster et $\mathrm{al}^{121}$ in a setting of $218 \mathrm{R} / \mathrm{R}$ B-NHL patients. Data from the dose-escalation part of the phase $\mathrm{I} / \mathrm{IB}$ study showed a positive toxicity profile and high efficacy in the subset of aNHLs, mainly represented by DLBCL $(n=87)$ and transformed FL $(n=29)$, with $64.1 \%$ and $42.2 \%$ ORR and CR, respectively. ${ }^{121}$ The study population was heavily pretreated with a median of three lines of therapy (range 1-14), including CAR-T in 23 of 218 cases. ORR and CR for patients $\mathrm{R} / \mathrm{R}$ to CAR- $\mathrm{T}$ were $43.8 \%$ and $25 \%$, respectively. Interestingly, after mosunetuzumab infusion, in vivo expansion of previously administered CAR-T cells was detected. ${ }^{121}$ Moreover, preliminary data suggest possible efficacy of retreatment with mosunetuzumab for nonresponders. ${ }^{121}$ Recently, an interim analysis of the GO40515 study, which investigated mosunetuzumab $+\mathrm{CHOP}$ in $\mathrm{R} / \mathrm{R} \mathrm{B}$ NHL and untreated DLBCL at high risk, has been presented. ${ }^{122}$ Manageable toxicity has been described, with CRS not exceeding grade 2 and no neurotoxicity observed. ${ }^{122}$ Among 27 DLBCL patients with evaluable response, an ORR of $96 \%$ was obtained with $85 \%$ CR. ${ }^{122}$ The safety and efficacy of mosunetuzumab as consolidation for patients affected by DLBCL with SD or PR after one line of therapy or mosunetuzumab \pm polatuzumab as frontline treatment for elderly/unfit DLBCL not considered candidates for immunochemotherapy are under investigation in a phase I/II trial. Preliminary data from the trial demonstrate acceptable toxicity and good rates of response of mosunetuzumab monotherapy in untreated unfit patients 
(cohort B): 58\% ORR and 42\% CR among different doses tested, with no grade 3 CRS or neurotoxicity observed. ${ }^{123,124}$

\section{Epcoritamab}

Epcoritamab (GEN3013) is another molecule belonging to the group of $\mathrm{CD} 3 \mathrm{xCD} 20$ bsAbs. It represents the first subcutaneous compound of this category and is characterized by binding the CD20 antigen on a different epitope to typical antiCD20 mAbs (ie, rituximab and GA101). Preliminary data of an ongoing phase $I / I I$ trial on $R / R$ B-NHLs have been presented, confirming manageable toxicity of this category of molecules, with 58\% CRS not exceeding grade 2 and $6 \%$ neurologic events (3\% grade 3 ), all transient. ${ }^{125,126}$ Epcoritamab demonstrated high activity on heavily pretreated B-NHL: in the dose-escalation part of the ongoing phase I/II study, among $45 \mathrm{R} / \mathrm{R}$ DLBCLs, including cases previously treated with CAR-T, an ORR of $66.7 \%$ (CR 33.3\%) for patients receiving epcoritamab $\geq 12 \mathrm{mg}$ and $100 \%$ (CR 28.6\%) for those receiving $\geq 48 \mathrm{mg}$ have been observed. ${ }^{126}$ Due to the promising antilymphoma activity of single-agent epcoritamab, a number of combinations with immunochemotherapy regimens, such as $\mathrm{R}-\mathrm{CHOP}, \mathrm{R}-\mathrm{GemOx}, \mathrm{BR}, \mathrm{R}^{2}$, rituximab + dexamethasone, cytarabine, and cisplatin/oxaliplatin (R-DHAP/X), are under investigation in phase I/II and III trials for both untreated and R/R FL and DLBCL. ${ }^{127,128}$

\section{Other Compounds}

Odronextamab and plamotamab represent two further CD3xCD20 mAbs tested on B-cell malignancies.

Odronextamab is a fully human $\mathrm{IgG}_{4}$-based bsAb, investigated in a phase I trial on $\mathrm{R} / \mathrm{R} \mathrm{B}-\mathrm{NHL}$, including FL and aNHL (mostly DLBCL). ${ }^{129129129129130}$ It showed acceptable toxicity and relevant antitumor activity, even at low doses ( $\geq 5 \mathrm{mg}$ ) on FL (ORR 92.9\%, CR 75\%), and when administered at higher doses $(\geq 80 \mathrm{mg}$ ) on DLBCLs (ORR and CR 60\%). A global phase II study for $\mathrm{R} / \mathrm{R}$ B-NHLs is ongoing.

Plamotamab is under investigation in a first-in-human trial for R/R B-NHLs and chronic lymphocytic leukemia (CLL). The trial is ongoing, and preliminary safety and antitumor-activity data on 44 subjects (36 B-NHLs, 8 CLL) are promising. ${ }^{131}$

\section{Conclusion}

MoAbs represent a major step forward in treatment of B-NHL, including LBCL, and are continuously improving survival for patients affected by these disorders, both as monotherapy and in combination with multiple chemotherapy regimens. Among naked mAbs, the novel anti-CD19 tafasitamab is the most promising agent, thanks to its synergic effect when combined with lenalidomide for $\mathrm{R} /$ $\mathrm{R}$ patients and the advantage of acting on an alternative target in respect of CD20. It received accelerated approval from the FDA in August 2020, and European approval is expected shortly, making tafasitamab in combination with lenalidomide already available in the US for the treatment of $\mathrm{R} / \mathrm{R}$ DLBCL.

In the field of ADCs, polatuzumab vedotin is gaining importance for $\mathrm{R} / \mathrm{R}$ DLBCL. The immunochemotherapy regimen pola-BR recently obtained approval for treatment of $R / R$ patients ineligible for or relapsed after ASCT or CAR-T, and it is now in clinical practice in many countries. Many other combinations of polatuzumab + chemotherapy are under investigation for LBCL, and we are particularly eager to learn of the results from the POLARIX trial to know if polatuzumab + R-CHOP can give an advantage over R-CHOP in frontline treatment.

Among LBCLs, anti-PD1/PDL1 checkpoint inhibitors have demonstrated effectiveness most mostly PMBCL. Nevertheless, emerging data on their capacity to enhance CAR-T efficacy is increasing interest in these compounds in the field of LBCL treatment.

The relatively recent discovery of the "do not eat me" tumor-immunoescape signal and its antagonism with antiCD47 compounds are surely of high interest. These drugs combined with rituximab and other agents could potentially have a role in the treatment of multirefractory patients, but further investigations are needed.

bsAbs represent a new frontier in therapeutic options for $\mathrm{R} / \mathrm{R}$ LBCL, showing promising results in phase $\mathrm{I} / \mathrm{II}$ trials both in terms of ORR and CR. Nowadays, they cannot be considered an alternative to CAR-T, due to a lack of consolidated long-term follow-up data. However, these compounds have the advantage of being an off-the-shelf option, more manageable (especially with subcutaneous administration), and with a safe toxicity profile when combined with chemotherapy, and they have now been moved to an earlier phase of treatment in LBCL. Based on results of combination studies, their characteristics may allow wider use of bsAbs in elderly and unfit patients, patients who have failed CART, or (in the not-too-distant future) first line in combination with standard immunochemotherapy in high-risk DLBCL patients. 
In future, the integration of multiple novel agents, including mAbs and other molecules, with RCHOP could increase the curable rate of frontline therapy for LBCL and increase the options of salvage regimens for $\mathrm{R} / \mathrm{R}$ patients.

\section{Author Contributions}

All authors made substantial contributions to conception and design, acquisition of data, or analysis and interpretation of data, took part in drafting the article or revising it critically for important intellectual content, agreed to submit to the current journal, gave final approval to the version to be published, and agree to be accountable for all aspects of the work.

\section{Funding}

No financial support was received for the production of this manuscript.

\section{Disclosure}

$\mathrm{U}$ Vitolo has acted on advisory boards for Janssen, Celgene, Genmab, Incyte, and Gilead, and has received lecture fees from Roche, Celgene, Janssen, AbbVie, and Gilead. The authors have no other relevant affiliations or financial involvement with any organization or entity with a financial interest in or conflict with the subject matter or materials discussed in this manuscript apart from those disclosed.

\section{References}

1. Swerdlow SH, Campo E, Pileri SA, et al. The 2016 revision of the World Health Organization classification of lymphoid neoplasms. Blood. 2016;127(20):2375-2390. doi:10.1182/blood-2016-01-643569

2. Sant M, Allemani $C$, Tereanu $C$, et al. Incidence of hematologic malignancies in Europe by morphologic subtype: results of the HAEMACARE project. Blood. 2010;116(19):3724-3734. doi:10. 1182/blood-2010-05-282632

3. Siegel RL, Miller KD, Jemal A. Cancer statistics, 2019. CA Cancer J Clin. 2019;69:7-34. doi:10.3322/caac.21551

4. Coiffier B, Lepage E, Brière J, et al. CHOP chemotherapy plus rituximab compared with $\mathrm{CHOP}$ alone in elderly patients with diffuse large-B-cell lymphoma. $N$ Engl $J$ Med. 2002;346:235-242. doi:10.1056/NEJMoa011795

5. Habermann TM, Weller EA, Morrison VA, et al. Rituximab-CHOP versus $\mathrm{CHOP}$ alone or with maintenance rituximab in older patients with diffuse large B-cell lymphoma. J Clin Oncol. 2006;24 (19):3121-3127. doi:10.1200/JCO.2005.05.1003

6. Pfreundschuh M, Trümper L, Österborg A, et al. CHOP-like chemotherapy plus rituximab versus $\mathrm{CHOP}$-like chemotherapy alone in young patients with good-prognosis diffuse large-B-cell lymphoma: a randomised controlled trial by the MabThera International Trial (MInT) Group. Lancet Oncol. 2006;7:379-391. doi:10.1016/S1470-2045(06)70664-7

7. Gisselbrecht C, Glass B, Mounier N, et al. Salvage regimens with autologous transplantation for relapsed large B-cell lymphoma in the rituximab era. J Clin Oncol. 2010;28(27):4184-4190. doi:10.1200/ JCO.2010.28.1618
8. Crump M, Neelapu SS, Farooq U, et al. Outcomes in refractory diffuse large B-cell lymphoma: results from the international SCHOLAR-1 study. Blood. 2017;130:1800-1808. doi:10.1182/ blood-2017-03-769620

9. Liu PP, Wang KF, Xia Y, et al. Racial patterns of patients with primary mediastinal large B-cell lymphoma. Med (United States). 2016;95:e4054.

10. Mary Dwyer N, Hema Sundar M, Fakhri B, et al. NCCN Guidelines Version 1.2021 B-cell lymphomas; 2021.

11. Vitolo U, Seymour JF, Martelli M, et al. Extranodal Diffuse large B-cell lymphoma (DLBCL) and primary mediastinal B-cell lymphoma: ESMO clinical practice guidelines for diagnosis, treatment and follow-up. Ann Oncol. 2016;27:v91-102. doi:10.1093/annonc/mdw175

12. Gleeson M, Hawkes EA, Cunningham D, et al. Rituximab, cyclophosphamide, doxorubicin, vincristine and prednisolone (R-CHOP) in the management of primary mediastinal B-cell lymphoma: a subgroup analysis of the UK NCRI R-CHOP 14 versus 21 trial. Br J Haematol. 2016;175:668-672. doi:10.1111/bjh.14287

13. Giulino-Roth L, O'Donohue T, Chen Z, et al. Outcomes of adults and children with primary mediastinal B-cell lymphoma treated with dose-adjusted EPOCH-R. $B r \quad J$ Haematol. 2017;179 (5):739-747. doi:10.1111/bjh.14951

14. Dunleavy K, Pittaluga S, Maeda LS, et al. Dose-adjusted EPOCH-rituximab therapy in primary mediastinal B-cell lymphoma. $N$ Engl J Med. 2013;368(15):1408-1416. doi:10. 1056/NEJMoa1214561

15. Moskowitz CH, Schöder H, Teruya-Feldstein J, et al. Risk-adapted dose-dense immunochemotherapy determined by interim FDG-PET in advanced-stage diffuse large B-cell lymphoma. J Clin Oncol. 2010;28(11):1896-1903. doi:10.1200/JCO.2009.26.5942

16. Zinzani PL, Stefoni V, Finolezzi E, et al. Rituximab combined with MACOP-B or VACOP-B and radiation therapy in primary mediastinal large B-cell lymphoma: a retrospective study. Clin Lymphoma Myeloma. 2009;9(5):381-385. doi:10.3816/CLM.2009.n.074

17. Savage KJ, Al-Rajhi N, Voss N, et al. Favorable outcome of primary mediastinal large B-cell lymphoma in a single institution: the British Columbia experience. Ann Oncol. 2006;17 (1):123-130. doi:10.1093/annonc/mdj030

18. Rieger M, Österborg A, Pettengell R, et al. Primary mediastinal B-cell lymphoma treated with CHOP-like chemotherapy with or without rituximab: results of the Mabthera International Trial Group study. Ann Oncol. 2011;22(3):664-670. doi:10.1093/ annonc/mdq418

19. Dunleavy K, Wilson WH. Primary mediastinal B-cell lymphoma and mediastinal gray zone lymphoma: do they require a unique therapeutic approach? Blood. 2015;125(1):33-39. doi:10.1182/ blood-2014-05-575092

20. Martelli M, Ferreri A, Di Rocco A, Ansuinelli M, Johnson PWM. Primary mediastinal large B-cell lymphoma. Crit Rev Oncol Hematol. 2017;113:318-327. doi:10.1016/j.critrevonc.2017.01.009

21. Rogers LM, Veeramani S, Weiner GJ. Complement in monoclonal antibody therapy of cancer. Immunol Res. 2014;59:203-210. doi:10.1007/s12026-014-8542-z

22. Maloney DG, Grillo-Ló Pez AJ, White CA, et al. IDEC-C2B8 (Rituximab) Anti-CD20 monoclonal antibody therapy in patients with relapsed low-grade non-Hodgkin's lymphoma. Blood. 1997;90:2188-2195. doi:10.1182/blood.V90.6.2188

23. Davies A, Berge C, Boehnke A, et al. Subcutaneous rituximab for the treatment of B-cell hematologic malignancies: a review of the scientific rationale and clinical development. $A d v$ Ther. 2017;34:2210-2231. doi:10.1007/s12325-017-0610-Z

24. Morschhauser FA, Cartron G, Thieblemont C, et al. Obinutuzumab (GA101) monotherapy in relapsed/refractory diffuse large b-cell lymphoma or mantle-cell lymphoma: results from the phase II GAUGUIN study. $J$ Clin Oncol. 2013;31:2912-2919. doi:10.1200/JCO.2012.46.9585 
25. Vitolo U, Trneny M, Belada D, et al. Obinutuzumab or rituximab plus cyclophosphamide, doxorubicin, vincristine, and prednisone in previously untreated diffuse large b-cell lymphoma. J Clin Oncol. 2017;35:3529-3537. doi:10.1200/JCO.2017.73.3402

26. Sehn LH, Martelli M, Trněný M, et al. A randomized, open-label, Phase III study of obinutuzumab or rituximab plus CHOP in patients with previously untreated diffuse large B-Cell lymphoma: final analysis of GOYA. J Hematol Oncol. 2020;13. doi:10.1186/s13045-020-00900-7

27. Merli F, Cavallo F, Salvi F, et al. Obinutuzumab and miniCHOP for unfit patients with diffuse large B-cell lymphoma. A phase II study by Fondazione Italiana Linfomi. $J$ Geriatr Oncol. 2020;11:37-40. doi:10.1016/j.jgo.2019.06.020

28. Horton HM, Bernett MJ, Pong E, et al. Potent in vitro and in vivo activity of an Fc-engineered anti-CD19 monoclonal antibody against lymphoma and leukemia. Cancer Res. 2008;68 (19):8049-8057. doi:10.1158/0008-5472.CAN-08-2268

29. Jurczak W, Zinzani PL, Gaidano G, et al. Phase IIa study of the CD19 antibody MOR208 in patients with relapsed or refractory B-cell non- Hodgkin's lymphoma. Ann Oncol. 2018;29:12 66-1272. doi:10.1093/annonc/mdy056

30. Awan FT, Lapalombella R, Trotta R, et al. CD19 targeting of chronic lymphocytic leukemia with a novel Fc-domainengineered monoclonal antibody. Blood. 2010;115:1204-1213. doi:10.1182/blood-2009-06-229039

31. Salles G, Duell J, González Barca E, et al. Tafasitamab plus lenalidomide in relapsed or refractory diffuse large B-cell lymphoma (L-MIND): a multicentre, prospective, single-arm, Phase 2 study. Lancet Oncol. 2020;21:978-988. doi:10.1016/S14702045(20)30225-4

32. Morschhauser F, Illidge T, Huglo D, et al. Efficacy and safety of yttrium-90 ibritumomab tiuxetan in patients with relapsed or refractory diffuse large B-cell lymphoma not appropriate for autologous stem-cell transplantation. Blood. 2007;110:54-58. doi:10.1182/blood-2007-01-068056

33. Ciochetto C, Botto B, Passera R, et al. Yttrium-90 ibritumomab tiuxetan (Zevalin) followed by BEAM (Z-BEAM) conditioning regimen and autologous stem cell transplantation (ASCT) in relapsed or refractory high-risk B-cell non-Hodgkin lymphoma (NHL): a single institution Italian experience. Ann Hematol. 2018;97:1619-1626. doi:10.1007/s00277-018-3328-3

34. Palanca-Wessels MCA, Czuczman M, Salles G, et al. Safety and activity of the anti-CD79B antibody-drug conjugate polatuzumab vedotin in relapsed or refractory B-cell non-Hodgkin lymphoma and chronic lymphocytic leukaemia: a Phase 1 study. Lancet Oncol. 2015;16:704-715. doi:10.1016/S1470-2045(15)70128-2

35. Advani RH, Lebovic D, Chen A, et al. Phase i study of the anti-CD22 antibody-drug conjugate pinatuzumab vedotin with/ without rituximab in patients with relapsed/refractory B-cell non-Hodgkin lymphoma. Clin Cancer Res. 2017;23:1167-1176. doi:10.1158/1078-0432.CCR-16-0772

36. Morschhauser F, Flinn IW, Advani R, et al. Polatuzumab vedotin or pinatuzumab vedotin plus rituximab in patients with relapsed or refractory non-Hodgkin lymphoma: final results from a phase 2 randomised study (ROMULUS). Lancet Haematol. 2019;6:e254 65. doi:10.1016/S2352-3026(19)30026-2

37. Tilly H, Morschhauser F, Bartlett NL, et al. Polatuzumab vedotin in combination with immunochemotherapy in patients with previously untreated diffuse large B-cell lymphoma: an open-label, non-randomised, phase 1b-2 study. Lancet Oncol. 2019;20:998-1010. doi:10.1016/S1470-2045(19)30091-9

38. Tilly H, Flowers C, Friedberg JW, et al. POLARIX: a phase 3 study of polatuzumab vedotin (pola) plus R-CHP versus R-CHOP in patients (pts) with untreated DLBCL. J Clin Oncol. 2019;37 (15_suppl):TPS7571. doi:10.1200/JCO.2019.37.15_suppl.TPS7571
39. Haioun C, Matasar MJ, Sancho J-M, et al. POLARGO: randomized Phase III study of polatuzumab vedotin plus rituximab, gemcitabine, and oxaliplatin (R-GemOx) in relapsed/refractory diffuse large B-cell lymphoma (R/R DLBCL). J Clin Oncol. 2020;38 (15_suppl):TPS8070. doi:10.1200/JCO.2020.38.15_suppl.TPS8070

40. Polatuzumab vedotin, rituximab, ifosfamide, carboplatin, and etoposide (PolaR-ICE) as initial salvage therapy for the treatment of relapsed/refractory diffuse large B-cell lymphoma Full Text View - ClinicalTrials.gov. Available from: https:// clinicaltrials.gov/ct2/show/NCT04665765. Accessed January 24, 2021.

41. Zammarchi F, Corbett S, Adams L, et al. ADCT-402, a PBD dimer-containing antibody drug conjugate targeting CD19expressing malignancies. Blood. 2018;131(10):1094-1105. doi:10.1182/blood-2017-10-813493

42. Hamadani M, Radford J, Carlo-Stella C, et al. Final results of a Phase 1 Study of loncastuximab tesirine in relapsed/refractory B-cell non-Hodgkin lymphoma. Blood. 2020. doi:10.1182/ blood.2020007512

43. Ribrag V, Dupuis J, Tilly H, et al. A dose-escalation study of SAR3419, an anti-CD19 antibody maytansinoid conjugate, administered by intravenous infusion once weekly in patients with relapsed/refractory B-cell non-Hodgkin lymphoma. Clin Cancer Res. 2014;20:213-220. doi:10.1158/1078-0432.CCR-13-0580

44. Younes A, Kim S, Romaguera J, et al. Phase I multidose-escalation study of the anti-CD19 maytansinoid immunoconjugate SAR3419 administered by intravenous infusion every 3 weeks to patients with relapsed/refractory B-cell lymphoma. $J$ Clin Oncol. 2012;30:2776-2782. doi:10.1200/JCO.2011.39.4403

45. Trněny M, Verhoef G, Dyer MJS, et al. A phase ii multicenter study of the anti-CD19 antibody drug conjugate coltuximab ravtansine (SAR3419) in patients with relapsed or refractory diffuse large B-cell lymphoma previously treated with rituximab-based immunotherapy. Haematologica. 2018;103:1351-1358. doi:10.33 24/haematol.2017.168401

46. Coiffier B, Thieblemont C, de Guibert S, et al. A Phase II, single-arm, multicentre study of coltuximab ravtansine (SAR3419) and rituximab in patients with relapsed or refractory diffuse large B-cell lymphoma. $B r \quad J$ Haematol. 2016;17 3:722-730. doi:10.1111/bjh.13992

47. Zinzani PL, Pellegrini C, Chiappella A, et al. Brentuximab vedotin in relapsed primary mediastinal large B-cell lymphoma: results from a phase 2 clinical trial. Blood. 2017;129(16):2328-2330. doi:10.1182/blood-2017-01-764258

48. Zinzani PL, Santoro A, Gritti G, et al. Nivolumab combined with brentuximab vedotin for relapsed/refractory primary mediastinal large B-cell lymphoma: efficacy and safety from the phase II checkmate 436 study. J Clin Oncol. 2019;37(33):3081-3089. doi:10.1200/JCO.19.01492

49. Jacobsen ED, Sharman JP, Oki Y, et al. Brentuximab vedotin demonstrates objective responses in a phase 2 study of relapsed/ refractory DLBCL with variable CD30 expression. Blood. 2015;125(9):1394-1402. doi:10.1182/blood-2014-09-598763

50. Svoboda J, Bair SM, Landsburg DJ, et al. Brentuximab vedotin in combination with rituximab, cyclophosphamide, doxorubicin, and prednisone as frontline treatment for patients with CD30-positive B-cell lymphomas. Haematologica. 2020:haematol.2019.238675. doi:10.3324/haematol.2019.238675

51. Brentuximab vedotin plus lenalidomide and rituximab for the treatment of relapsed/refractory DLBCL - Full Text View ClinicalTrials.gov. Available from: https://www.clinicaltrials.gov/ ct2/show/NCT04404283. Accessed January 31, 2021.

52. Schaper F, van Spriel AB. Antitumor immunity is controlled by tetraspanin proteins. Front Immunol. 2018;9:1185. doi:10.3389/ fimmu.2018.01185 
53. Xu-Monette ZY, Li L, Byrd JC, et al. Assessment of CD37 B-cell antigen and cell of origin significantly improves risk prediction in diffuse large B-cell lymphoma. Blood. 2016;128:3083-3100. doi:10.1182/blood-2016-05-715094

54. Stathis A, Flinn IW, Madan S, et al. Safety, tolerability, and preliminary activity of IMGN529, a CD37-targeted antibody-drug conjugate, in patients with relapsed or refractory B-cell non-Hodgkin lymphoma: a dose-escalation, phase I study. Invest New Drugs. 2018;36:869-876. doi:10.1007/s10637-018-0570-4

55. Study to evaluate the efficacy and tolerability Debio 1562 in combination with rituximab in patients with relapsed and/or refractory DLBCL and other forms of NHL - Full Text View ClinicalTrials.gov. Available from: https://clinicaltrials.gov/ct2/ show/NCT02564744. Accessed April 10, 2021.

56. Kroschinsky F, Middeke JM, Janz M, et al. Phase I dose escalation study of BI 836826 (CD37 antibody) in patients with relapsed or refractory B-cell non-Hodgkin lymphoma. Invest New Drugs. 2020;38(5):1472-1482. doi:10.1007/s10637-020-00916-3

57. Shimoni A, Avivi I, Rowe JM, et al. A randomized study comparing yttrium-90 ibritumomab tiuxetan (Zevalin) and high-dose BEAM chemotherapy versus BEAM alone as the conditioning regimen before autologous stem cell transplantation in patients with aggressive lymphoma. Cancer. 2012;118:4706-4714. doi:10. $1002 /$ cncr. 27418

58. Sehn LH, Herrera AF, Flowers CR, et al. Polatuzumab vedotin in relapsed or refractory diffuse large B-cell lymphoma. J Clin Oncol. 2020;38(2):155-165. doi:10.1200/JCO.19.00172

59. Pereira DS, Guevara CI, Jin L, et al. AGS67E, an anti-CD37 monomethyl auristatin E antibody-drug conjugate as a potential therapeutic for B/T-cell malignancies and AML: a new role for CD37 in AML. Mol Cancer Ther. 2015;14:1650-1660. doi:10.1158/1535-7163.MCT-15-0067

60. Robak T, Hellmann A, Kloczko J, et al. Randomized phase 2 study of otlertuzumab and bendamustine versus bendamustine in patients with relapsed chronic lymphocytic leukaemia. $\mathrm{Br}$ J Haematol. 2017;176:618-628. doi:10.1111/bjh.14464

61. A study to evaluate safety, tolerability, and pharmacokinetics of escalating doses of ags67e given as monotherapy in subjects with refractory or relapsed lymphoid malignancies - Full Text View ClinicalTrials.gov. Available from: https://clinicaltrials.gov/ct2/ show/NCT02175433. Accessed April 10, 2021.

62. Lesokhin AM, Ansell SM, Armand P, et al. Nivolumab in patients with relapsed or refractory hematologic malignancy: preliminary results of a phase ib study. J Clin Oncol. 2016;34:2698-2704. doi:10.1200/JCO.2015.65.9789

63. Ansell SM, Minnema MC, Johnson P, et al. Nivolumab for relapsed/refractory diffuse large B-cell lymphoma in patients ineligible for or having failed autologous transplantation: a single-arm, phase II study. J Clin Oncol. 2019;37:481-489. doi:10.1200/JCO.18.00766

64. Cao Y, Lu W, Sun R, et al. Anti-CD19 chimeric antigen receptor $\mathrm{T}$ cells in combination with nivolumab are safe and effective against relapsed/refractory B-cell non-Hodgkin lymphoma. Front Oncol. 2019;9. doi:10.3389/fonc.2019.00767

65. Bond DA, Yildiz V, Wei L, et al. A Phase I study of nivolumab and lenalidomide in relapsed/refractory B cell lymphoma. Blood. 2019;134:4091. doi:10.1182/blood-2019-130539

66. Nivolumab with or without varlilumab in treating patients with relapsed or refractory aggressive B-cell lymphomas - Full Text View - ClinicalTrials.gov. Available from: https:/clinicaltrials. gov/ct2/show/NCT03038672. Accessed February 21, 2021.

67. Nivolumab/ipilimumab-primed immunotransplant for DLBCL Full Text View - ClinicalTrials.gov. Available from: https://clin icaltrials.gov/ct2/show/NCT03305445. Accessed February 21, 2021.
68. Nivolumab and combination chemotherapy in treating participants with diffuse large B-cell lymphoma - Full Text View ClinicalTrials.gov. Available from: https://clinicaltrials.gov/ct2/ show/NCT03704714. Accessed February 21, 2021.

69. Safety and efficacy of bendamustine, gemcitabine, rituximab, nivolumab (BeGeRN) in patients with $\mathrm{r} / \mathrm{r}$ DLBCL - Full Text View - ClinicalTrials.gov. Available from: https://clinicaltrials. gov/ct2/show/NCT03259529. Accessed February 21, 2021.

70. Rituximab, lenalidomide, and nivolumab in treating participants with relapsed or refractory non-germinal center type diffuse large B cell lymphoma or primary central nervous system lymphoma - Full Text View - ClinicalTrials.gov. Available from: https:/clinicaltrials.gov/ct2/ show/NCT03558750. Accessed February 21, 2021.

71. A study of the safety, tolerability, and efficacy of epacadostat administered in combination with nivolumab in select advanced cancers (ECHO-204) - Full Text View - ClinicalTrials.gov. Available from: https://clinicaltrials.gov/ct2/show/ NCT02327078. Accessed February 21, 2021.

72. Zinzani PL, Ribrag V, Moskowitz CH, et al. Safety and tolerability of pembrolizumab in patients with relapsed/refractory primary mediastinal large B-cell lymphoma. Blood. 2017;130 (3):267-270. doi:10.1182/blood-2016-12-758383

73. Armand P, Rodig S, Melnichenko V, et al. Pembrolizumab in relapsed or refractory primary mediastinal large b-cell lymphoma. J Clin Oncol. 2019;37(34):3291-3299. doi:10.1200/ JCO.19.01389

74. Sheikh S, Kuruvilla J. Pembrolizumab for the treatment of diffuse large B-cell lymphoma. Expert Opin Biol Ther. 2019;19 (11):1119-1126. doi:10.1080/14712598.2019.1659777

75. Godfrey J, Tumuluru S, Bao R, et al. PD-L1 gene alterations identify a subset of diffuse large B-cell lymphoma harboring a T-cell-inflamed phenotype. Blood. 2019;133(21):2279-2290. doi:10.1182/blood-2018-10-879015

76. Andorsky DJ, Yamada RE, Said J, Pinkus GS, Betting DJ, Timmerman JM. Programmed death ligand 1 is expressed by non-Hodgkin lymphomas and inhibits the activity of tumor-associated T cells. Clin Cancer Res. 2011;17:4232-4244. doi:10.1158/1078-0432.CCR-10-2660

77. Pembrolizumab for patients with PD-L1 diffuse large B cell lymphoma (DLBCL) - Full Text View - ClinicalTrials.gov. Available from: https://clinicaltrials.gov/ct2/show/NCT03990961. Accessed February 21, 2021.

78. Herrera AF, Chen L, Popplewell LL, et al. Preliminary results from a Phase I trial of pembrolizumab plus vorinostat in patients with relapsed or refractory diffuse large B-cell lymphoma, follicular lymphoma, and Hodgkin lymphoma. Blood. 2019;134 (Supplement_1):759. doi:10.1182/blood-2019-123163

79. Smith SD, Till BG, Shadman MS, et al. Pembrolizumab with RCHOP in previously untreated diffuse large B-cell lymphoma: potential for biomarker driven therapy. Br J Haematol. 2020;189:1119-1126. doi:10.1111/bjh.16494

80. Chong EA, Melenhorst JJ, Lacey SF, et al. PD-1 blockade modulates chimeric antigen receptor (CAR)-modified T cells: refueling the CAR. Blood. 2017;129:1039-1041. doi:10.1182/ blood-2016-09-738245

81. Chong EA, Svoboda J, Dwivedy Nasta S, et al. Sequential anti-CD19 directed chimeric antigen receptor modified T-cell therapy (CART19) and PD-1 blockade with pembrolizumab in patients with relapsed or refractory B-cell non-Hodgkin lymphomas. Blood. 2018;132(Supplement 1):4198. doi:10.1182/ blood-2018-99-119502

82. Jaeger U, Worel N, McGuirk JP, et al. Portia: a Phase 1b study evaluating safety and efficacy of tisagenlecleucel and pembrolizumab in patients with relapsed/refractory diffuse large B-cell lymphoma. Blood. 2019;134:5325. doi:10.1182/blood-2019-129120 
83. Osborne W, Marzolini M, Tholouli E, et al. Phase I Alexander study of AUTO3, the first CD19/22 dual targeting CAR T cell therapy, with pembrolizumab in patients with relapsed/refractory (r/r) DLBCL. J Clin Oncol. 2020;38:8001. doi:10.1200/JCO.20 20.38.15_suppl.8001

84. Frigault MJ, Armand P, Redd RA, et al. PD-1 blockade for diffuse large B-cell lymphoma after autologous stem cell transplantation. Blood Adv. 2020;4:122-126. doi:10.1182/bloodadvances.2019 000784

85. Open label study investigating the safety and efficacy of blinatumomab in combination with pembrolizumab (KEYNOTE-348) Full Text View - ClinicalTrials.gov. Available from: https://clin icaltrials.gov/ct2/show/NCT03340766. Accessed February 21, 2021.

86. Joffe E, Vardhana SA, Kumar A, et al. A phase I and randomized phase II etctn study of KW-0761 (Mogamulizumab) and MK-3475 (Pembrolizumab) in relapsed and refractory diffuse large B-cell lymphoma. J Clin Oncol. 2020;38(15_suppl): TPS8072. doi:10.1200/JCO.2020.38.15_suppl.TPS8072

87. Younes A, Burke JM, Cheson BD, et al. Safety and efficacy of atezolizumab in combination with rituximab plus CHOP in previously untreated patients with diffuse large B-Cell Lymphoma (DLBCL): updated analysis of a Phase I/II Study. Blood. 2019;134:2874. doi:10.1182/blood-2019-123368

88. Herbaux C, Casasnovas O, Feugier P, et al. Atezolizumab + obinutuzumab + venetoclax in patients with relapsed or refractory diffuse large B-cell Lymphomas (R/R DLBCL): primary analysis of a phase II trial from LYSA. J Clin Oncol. 2020;38 (15_suppl):8053. doi:10.1200/JCO.2020.38.15_suppl.8053

89. Jacobson CA, Westin JR, Miklos DB, et al. Abstract CT055: phase 1/2 primary analysis of ZUMA-6: axicabtagene ciloleucel (Axi-Cel) in combination With atezolizumab (Atezo) for the treatment of patients (Pts) with refractory diffuse large B cell lymphoma (DLBCL). Cancer Res. 2020;(suppl):CT055-CT055.

90. Chen RW, Ansell SM, Zinzani PL, et al. Phase 1b/3 study of avelumab-based combination regimens in patients with relapsed or refractory diffuse large B-cell lymphoma (R/R DLBCL). J Clin Oncol. 2017;35(15_suppl):TPS7575. doi:10.1200/JCO.2017.35. 15_suppl.TPS7575

91. Hawkes EA, Chong G, Smith C, et al. Safety and efficacy of induction and maintenance avelumab plus R-CHOP in patients with diffuse large B-cell lymphoma (DLBCL): analysis of the Phase II Avr-CHOP Study. Blood. 2020;136:43-44. doi:10.1182/ blood-2020-136024

92. Avelumab, utomilumab, rituximab, ibrutinib, and combination chemotherapy in treating patients with relapsed or refractory diffuse large B-cell lymphoma or mantle cell lymphoma - Full Text View - ClinicalTrials.gov. Available from: https://clinical trials.gov/ct2/show/NCT03440567. Accessed February 21, 2021.

93. Casulo C, Santoro A, Ando K, et al. Durvalumab (Anti PD-L1) as monotherapy or in combination therapy for relapsed/refractory $(\mathrm{r} /$ r) diffuse large B-cell lymphoma (DLBCL) and Follicular Lymphoma (FL): a subgroup analysis from the Phase 1/2 Fusion NHL-001 Global Multicenter Trial. Blood. 2019;134:5320. doi:10.1182/blood-2019-124102

94. Nowakowski GS, Willenbacher W, Greil R, et al. Safety and efficacy of PD-L1 inhibitor durvalumab with R-CHOP or R 2 CHOP in subjects with previously untreated, high-risk DLBCL. J Clin Oncol. 2019;37:7520. doi:10.1200/JCO.2019.37.15_suppl. 7520

95. Ribrag V, Lee ST, Rizzieri D, et al. A Phase 1b Study to evaluate the safety and efficacy of durvalumab in combination with tremelimumab or danvatirsen in patients with relapsed or refractory diffuse large B-cell lymphoma. Clin Lymphoma Myeloma Leuk. 2020. doi:10.1016/j.clml.2020.12.012
96. Herrera AF, Goy A, Mehta A, et al. Safety and activity of ibrutinib in combination with durvalumab in patients with relapsed or refractory follicular lymphoma or diffuse large B-cell lymphoma. Am J Hematol. 2020;95:18-27. doi:10.1002/ ajh.25659

97. Hirayama AV, Gauthier J, Hay KA, et al. Efficacy and toxicity of JCAR014 in combination with durvalumab for the treatment of patients with relapsed/refractory aggressive B-cell non-Hodgkin lymphoma. Blood. 2018;132:1680. doi:10.1182/blood-2018-99-116745

98. Study of durvalumab and lenalidomide in R/R EBV associated DLBCL subtypes, primary CNS and testicular DLBCL - Full Text View - ClinicalTrials.gov. Available from: https:/clinical trials.gov/ct2/show/NCT03212807. Accessed February 21, 2021.

99. Powderly JD, Gutierrez M, Wang D, et al. A phase $1 \mathrm{~b} / 2$, open-label study to evaluate the safety and tolerability of MEDI6469 in combination with immune therapeutic agents or therapeutic mAbs in patients with selected advanced solid tumors or aggressive B-cell lymphomas. J Clin Oncol. 2015;33(suppl):TPS3091. doi:10.1200/jco.2015.33.15_suppl. tps3091

100. Durvalumab in DLBCL after autologous transplant - Full Text View - ClinicalTrials.gov. Available from: https://clinicaltrials. gov/ct2/show/NCT03241017. Accessed February 21, 2021.

101. Teft WA, Kirchhof MG, Madrenas J. A molecular perspective of CTLA-4 function. Annu Rev Immunol. 2006;24:65-97. doi:10.1146/annurev.immunol.24.021605.090535

102. Ansell SM, Hurvitz SA, Koenig PA, et al. Phase I study of ipilimumab, an anti-CTLA-4 monoclonal antibody, in patients with relapsed and refractory B-cell non-Hodgkin lymphoma. Clin Cancer Res. 2009;15:6446-6453. doi:10.1158/1078-0432. CCR-09-1339

103. Tuscano JM, Maverakis E, Groshen S, et al. A phase I study of the combination of rituximab and ipilimumab in patients with relapsed/refractory B-cell lymphoma. Clin Cancer Res. 2019;25:7004-7013. doi:10.1158/1078-0432.CCR-19-0438

104. Skarbnik AP, Donato ML, Feinman R, et al. Safety and efficacy of consolidation therapy with ipilimumab plus nivolumab after autologous stem cell transplantation. Transplant Cell Ther. 2020. doi:10.1016/j.jtct.2020.12.026

105. Tsai RK, Discher DE. Inhibition of 'self' engulfment through deactivation of myosin-II at the phagocytic synapse between human cells. J Cell Biol. 2008;180:989-1003. doi:10.1083/ jcb.200708043

106. Majeti R, Chao MP, Alizadeh AA, et al. CD47 is an adverse prognostic factor and therapeutic antibody target on human acute myeloid leukemia stem cells. Cell. 2009;138:286-299. doi:10.1016/j.cell.2009.05.045

107. Advani R, Flinn I, Popplewell L, et al. CD47 blockade by Hu5F9G4 and rituximab in non-Hodgkin's lymphoma. $N$ Engl J Med. 2018;379:1711-1721. doi:10.1056/NEJMoa1807315

108. Advani R, Bartlett NL, Smith SM, et al. The first-in-class anti-CD47 antibody HU5F9-G4 + rituximab induces durable responses in relapsed/refractory DLBCL and indolent lymphoma: interim phase 1b/2 results. Hematol Oncol. 2019;37:89-90. doi:10.1002/hon.57_2629

109. Ansell SM, Maris M, Lesokhin AM, et al. Phase 1 Study of the CD47 blocker TTI-621 in patients with relapsed or refractory hematologic malignancies. Clin Cancer Res. 2021;27(8):21 90-2199. doi:10.1158/1078-0432.CCR-20-3706

110. Goebeler ME, Knop S, Viardot A, et al. Bispecific T-cell engager (BiTE) antibody construct Blinatumomab for the treatment of Patients with relapsed/refractory non-Hodgkin lymphoma: final results from a phase I study. J Clin Oncol. 2016;34:1104-1111. doi:10.1200/JCO.2014.59.1586 
111. Viardot A, Goebeler ME, Hess G, et al. Phase 2 study of the bispecific T-cell engager (bite) antibody Blinatumomab in relapsed/refractory diffuse large B-cell lymphoma. Blood. 2016;127:1410-1416. doi:10.1182/blood-2015-06-651380

112. Study to evaluate safety and efficacy of blinatumomab in subjects with relapsed/refractory (R/R) aggressive B-cell NHL - Full Text View - ClinicalTrials.gov. Available from: https://clinicaltrials. gov/ct2/show/NCT02910063. Accessed February 7, 2021.

113. Hutchings M, Iacoboni G, Morschhauser F, et al. CD20-Tcb (RG6026), a Novel '2:1' format T-cell-engaging bispecific antibody, induces complete remissions in relapsed/refractory B-cell Non-Hodgkin's lymphoma: preliminary results from a Phase I First in Human Trial. Blood. 2018;132:226. doi:10.1182/blood2018-99-110207

114. Bacac M, Colombetti S, Herter S, et al. CD20-TCB with obinutuzumab pretreatment as next-generation treatment of hematologic malignancies. Clin Cancer Res. 2018;24:4785-4797. doi:10.1158/1078-0432.CCR-18-0455

115. Dickinson MJ, Morschhauser F, Iacoboni G, et al. CD20-TCB in relapsed or refractory non-Hodgkin lymphoma: durable complete responses and manageable safety observed at clinically relevant doses in phase I dose escalation. EHA Libr. 2020;4(suppl):S241.

116. Morschhauser F, Carlo-Stella C, Offner F, et al. Dual CD20-targeted therapy with concurrent CD20-TCB and obinutuzumab shows highly promising clinical activity and manageable safety in relapsed or refractory B-cell Non-Hodgkin lymphoma: preliminary results from a Phase Ib Trial. Blood. 2019;134:1584. doi:10.1182/blood-2019-123949

117. An open-label Phase 1B Study of glofitamab and atezolizumab or polatuzumab vedotin in adult patients with relapsed/refractory B-cell non-Hodgkin's Lymphoma - Full Text View ClinicalTrials.gov. Available from: https://clinicaltrials.gov/ct2/ show/NCT03533283. Accessed February 7, 2021.

118. Hutchings M, Gritti G, Sureda A, et al. CD20-TCB, a novel T-cell-engaging bispecific antibody, can be safely combined with the anti-PD-L1 antibody atezolizumab in relapsed or refractory B-cell non-Hodgkin lymphoma. Blood. 2019;134:2871. doi:10.1182/blood-2019-123978

119. A Phase III study evaluating glofitamab in combination with gemcitabine + oxaliplatin vs rituximab in combination with gemcitabine + oxaliplatin in participants with relapsed/refractory diffuse large B-cell lymphoma - Full Text View - ClinicalTrials.gov. Available from: https://clinicaltrials.gov/ct2/show/NCT04408638. Accessed February 7, 2021

120. A study of glofitamab in combination with rituximab or obinutuzumab plus cyclophosphamide, doxorubicin, vincristine, and prednisone (CHOP) in participants with non-Hodgkin lymphomas and participants with DLBCL - Full Text View - ClinicalTrials. gov. Available from: https://clinicaltrials.gov/ct2/show/ NCT03467373. Accessed February 7, 2021.

121. Schuster SJ, Bartlett NL, Assouline S, et al. Mosunetuzumab induces complete remissions in poor prognosis non-Hodgkin lymphoma patients, including those who are resistant to or relapsing after chimeric antigen receptor T-cell (CAR-T) therapies, and is active in treatment through multiple lines. Blood. 2019;134:6. doi:10.1182/blood-2019-123742

Biologics: Targets and Therapy

\section{Publish your work in this journal}

Biologics: Targets and Therapy is an international, peer-reviewed journal focusing on the patho-physiological rationale for and clinical application of Biologic agents in the management of autoimmune diseases, cancers or other pathologies where a molecular target can be identified. This journal is indexed on PubMed Central, CAS, EMBase,
122. Phillips TJ, Olszewski AJ, Munoz J, et al. Mosunetuzumab, a Novel CD20/CD3 bispecific antibody, in combination with CHOP confers high response rates in patients with diffuse large B-cell lymphoma. Blood. 2020;136:37-38. doi:10.1182/blood2020-136295

123. Trial of mosunetuzumab (BTCT4465A) as consolidation therapy in participants with diffuse large B-cell lymphoma following first-line immunochemotherapy and as monotherapy or in combination with polatuzumab vedotin in elderly/unfit participants with previously untreated diffuse large B-cell lymphoma - Full Text View - ClinicalTrials.gov. Available from: https://clinicaltrials. gov/ct2/show/NCT03677154. Accessed February 13, 2021.

124. Olszewski AJ, Avigdor A, Babu S, et al. Single-agent mosunetuzumab is a promising safe and efficacious chemotherapy-free regimen for elderly/unfit patients with previously untreated diffuse large B-cell lymphoma. Blood. 2020;136(Supplement 1):43-45. doi:10.1182/blood-2020-136255

125. Hutchings $M$, Lugtenburg $P$, Mous $R$, et al. Epcoritamab (GEN3013; DuoBody-CD3 $\times$ CD20) to induce complete response in patients with relapsed/refractory B-cell non-Hodgkin lymphoma (B-NHL): complete dose escalation data and efficacy results from a phase I/II trial. J Clin Oncol. 2020;38:8009. doi:10.1200/JCO.2020.38.15_suppl.8009

126. Hutchings M, Mous R, Clausen MR, et al. Subcutaneous epcoritamab induces complete responses with an encouraging safety profile across relapsed/refractory B-cell non-Hodgkin lymphoma subtypes, including patients with prior CAR-T therapy: updated dose escalation data. Blood. 2020;136:45-46. doi:10.1182/blood2020-133820

127. Safety and efficacy trial of epcoritamab combinations in subjects with B-cell non-Hodgkin lymphoma - Full Text View ClinicalTrials.gov. Available from: https://clinicaltrials.gov/ct2/ show/NCT04663347. Accessed February 7, 2021.

128. A Phase 3 Trial of epcoritamab in R/R DLBCL - Full Text View ClinicalTrials.gov. Available from: https://clinicaltrials.gov/ct2/ show/NCT04628494. Accessed February 7, 2021.

129. Bannerji R, Allan JN, Arnason JE, et al. Odronextamab (REGN1979), a human CD20 x CD3 bispecific antibody, induces durable, complete responses in patients with highly refractory B-cell non-Hodgkin lymphoma, including patients refractory to CAR T therapy. Blood. 2020;136(Supplement 1):42-43. doi:10.1182/blood-2020-136659

130. Assess the anti-tumor activity and safety of odronextamab in patients with relapsed or refractory B-cell non-Hodgkin lymphoma - Full Text View - ClinicalTrials.gov. Available from: https://clinicaltrials.gov/ct2/show/NCT03888105. Accessed February 14, 2021.

131. Patel K, Michot J-M, Chanan-Khan AA, et al. Preliminary safety and anti-tumor activity of XmAb13676, an anti-CD20 x anti-CD3 bispecific antibody, in patients with relapsed/refractory nonHodgkin's lymphoma and chronic lymphocytic leukemia. Blood. 2019;134(Supplement_1):4079. doi:10.1182/blood-2019-128564
Scopus and the Elsevier Bibliographic databases. The manuscript management system is completely online and includes a very quick and fair peer-review system, which is all easy to use. Visit http://www.dovepress.com/testimonials.php to read real quotes from published authors. 